Document downloaded from:

http://hdl.handle.net/10251/120925

This paper must be cited as:

Behl, R.; Cordero Barbero, A.; Motsa, SS.; Torregrosa Sánchez, JR. (2018). Multiplicity anomalies of an optimal fourth-order class of iterative methods for solving nonlinear equations. Nonlinear Dynamics. 91(1):81-112. https://doi.org/10.1007/s11071-017-3858-6

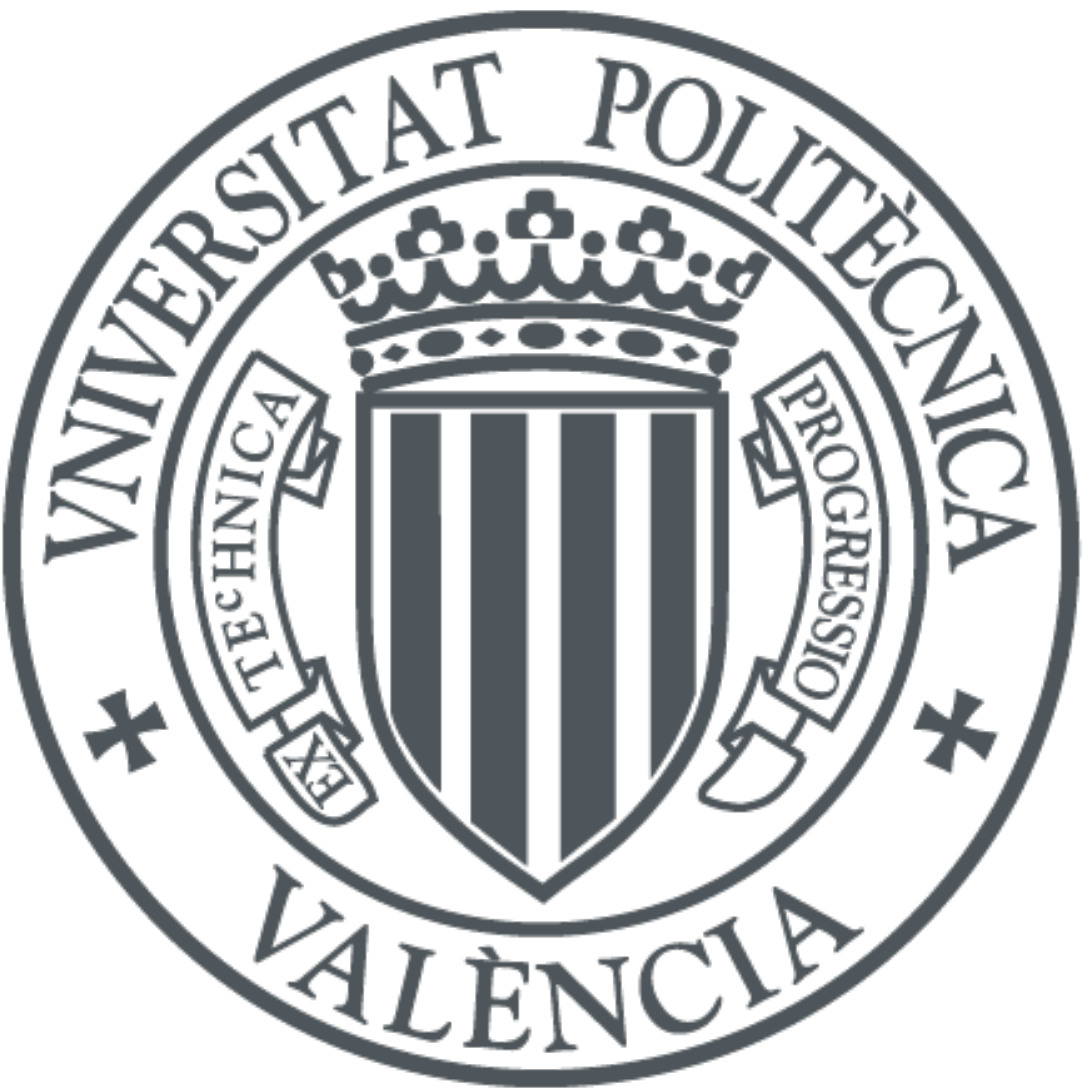

The final publication is available at

http://doi.org/10.1007/s11071-017-3858-6

Copyright Springer-Verlag

Additional Information 


\title{
Multiplicity anomalies of an optimal fourth-order class of iterative methods for solving nonlinear equations
}

\author{
Ramandeep Behl · Alicia Cordero · Sandile S. Motsa · Juan R. \\ Torregrosa
}

Received: date / Accepted: date

\begin{abstract}
There is a few number of optimal fourth-order iterative methods for obtaining the multiple roots of nonlinear equations. But, in most of the earlier studies, scholars gave the flexibility in their proposed schemes only at the second step (not at the first step) in order to explore new schemes. Unlike what happens in existing methods, the main aim of this manuscript is to construct a new fourth-order optimal scheme which will gives the flexibility to the researchers at both steps as well as faster convergence, smaller residual errors and asymptotic error constants. The construction of the proposed scheme is based on the mid-point formula and weight function approach. From the computational point of view, the stability of the resulting class of iterative methods is studied by means of the conjugacy maps and the analysis of strange fixed points. Their basins of attractions and parameter planes are also given to show their dynamical behavior around the multiple roots. Finally, we consider a real life problem and a concrete variety of standard test functions for numerical experiments and relevant results are extensively treated to confirm the theoretical development.
\end{abstract}

Keywords Nonlinear equations $\cdot$ Multiple roots $\cdot$ Complex dynamics $\cdot$ Dynamical and parameter plane $\cdot$ Stability

\section{Introduction}

High-order multipoint iterative schemes $[5,10,16]$ are always required for obtaining the multiple roots with multiplicity $m$ of a nonlinear equation $f(x)=0$, which partake of scientific, applied science, engineering and various other models. So, multi-point iterative methods overcome from the theoretical limitation of one-point iterative methods which concern about the order of convergence and computational efficiency. Further, they are also capable to generate approximated root of of higher accuracy with in a small number of iterations without requiring the evaluations of second-order derivatives.

This research was partially supported by Ministerio de Economía y Competitividad MTM2014-52016-C2-2-P and Generalitat Valenciana PROMETEO/2016/089.

Ramandeep Behl

School of Mathematical Sciences, University of KwaZulu-Natal,

Private Bag X01, Scottsville 3209, Pietermaritzburg, South Africa

Department of Mathematics, King Abdulaziz University,

Jeddah 21577, Saudi Arabia

E-mail: ramanbeh187@yahoo.in

Alicia Cordero

Instituto Universitario de Matemática Multidisciplinar

Universitat Politècnica de València, Spain

E-mail: acordero@mat.upv.es

Sandile S. Motsa

School of Mathematical Sciences, University of KwaZulu-Natal,

Private Bag X01, Scottsville 3209, Pietermaritzburg, South Africa

E-mail: sandilemotsa@gmail.com

Juan R.Torregrosa

Instituto Universitario de Matemática Multidisciplinar

Universitat Politècnica de València, Spain

E-mail: jrtorre@mat.upv.es 
In the text of Traub [16], we can find the well-known modification of Newton's method due to Schöder [12], with second order of convergence

$$
x_{n+1}=x_{n}-m \frac{f\left(x_{n}\right)}{f^{\prime}\left(x_{n}\right)}
$$

and also the sequence, designed by Traub, of iterative methods of increasing order of convergence. This order was independent of the known multiplicity $m$.

In 2009, Li et al. [9] presented the following optimal (according to Kung-Traub conjecture [7]) fourth-order multi-point iterative method

$$
\left\{\begin{aligned}
y_{n} & =x_{n}-\frac{2 m}{m+2} \frac{f\left(x_{n}\right)}{f^{\prime}\left(x_{n}\right)}, \\
x_{n+1} & =x_{n}-\frac{f\left(x_{n}\right)}{2 f^{\prime}\left(x_{n}\right)}\left[\frac{m(m-2)\left(\frac{m}{m+2}\right)^{-m} f^{\prime}\left(y_{n}\right)-m^{2} f^{\prime}\left(x_{n}\right)}{f^{\prime}\left(x_{n}\right)-\left(\frac{m}{m+2}\right)^{-m} f^{\prime}\left(y_{n}\right)}\right] .
\end{aligned}\right.
$$

This method was designed as a Jarratt-type scheme, whose coefficients depend on the multiplicity $m$, by using two functional evaluations of $f^{\prime}(x)$ and one of $f(x)$.

Again in 2010, Li et al. [8] gave a total number of six multi-point iterative methods of fourth-order with closed formulas for obtaining multiple roots. Out of them, only two were optimal, which are given as follows:

$$
\left\{\begin{array}{c}
y_{n}=x_{n}-\frac{2 m}{m+2} \frac{f\left(x_{n}\right)}{f^{\prime}\left(x_{n}\right)}, \\
x_{n+1}=x_{n}-\theta_{1} \frac{f\left(x_{n}\right)}{f^{\prime}\left(y_{n}\right)}-\frac{f\left(x_{n}\right)}{\theta_{2} f^{\prime}\left(x_{n}\right)+\theta_{3} f^{\prime}\left(y_{n}\right)},
\end{array}\right.
$$

where

$$
\begin{aligned}
& \theta_{1}=-\frac{1}{2} \frac{\left(\frac{m}{m+2}\right)^{m} m\left(m^{4}+4 m^{3}-16 m-16\right)}{m^{3}-4 m+8} \\
& \theta_{2}=-\frac{\left(m^{3}-4 m+8\right)^{2}}{m\left(m^{4}+4 m^{3}-4 m^{2}-16 m+16\right)\left(m^{2}+2 m-4\right)} \\
& \theta_{3}=\frac{m^{2}\left(m^{3}-4 m+8\right)}{\left(\frac{m}{m+2}\right)^{m}\left(m^{4}+4 m^{3}-4 m^{2}-16 m+16\right)\left(m^{2}+2 m-4\right)}
\end{aligned}
$$

and

$$
\left\{\begin{aligned}
y_{n} & =x_{n}-\frac{2 m}{m+2} \frac{f\left(x_{n}\right)}{f^{\prime}\left(x_{n}\right)} \\
x_{n+1} & =x_{n}-\theta_{4} \frac{f\left(x_{n}\right)}{f^{\prime}\left(x_{n}\right)}-\frac{f\left(x_{n}\right)}{\theta_{5} f^{\prime}\left(x_{n}\right)+\theta_{6} f^{\prime}\left(y_{n}\right)}
\end{aligned}\right.
$$

being

$$
\theta_{4}=-\frac{1}{2} m^{2}+m, \quad \theta_{5}=-\frac{1}{m}, \quad \theta_{6}=\frac{1}{m\left(\frac{m}{m+2}\right)^{m}} .
$$

Later, in 2011, Zhou et al. in [18], presented a more general optimal family of fourth-order iterative methods based on the weight function approach which require only three functional evaluations per iteration, which is defined as follows:

$$
\left\{\begin{array}{c}
y_{n}=x_{n}-\frac{2 m}{m+2} \frac{f\left(x_{n}\right)}{f^{\prime}\left(x_{n}\right)} \\
x_{n+1}=x_{n}-Q\left(\frac{f^{\prime}\left(y_{n}\right)}{f^{\prime}\left(x_{n}\right)}\right) \frac{f\left(x_{n}\right)}{f^{\prime}\left(x_{n}\right)}
\end{array}\right.
$$

where $Q(.) \in C^{2}(\mathbb{R})$ is a weight function. One can also obtain the expressions namely, expression (1), expression (6), expression (8), and expression (9) as special cases of scheme (4) just by considering suitable choice of weight functions.

In addition, in 2012, Sharifi et al. in [15], gave the following optimal scheme of fourth-order methods

$$
\left\{\begin{aligned}
y_{n} & =x_{n}-\frac{2 m}{m+2} \frac{f\left(x_{n}\right)}{f^{\prime}\left(x_{n}\right)}, \\
x_{n+1} & =x_{n}+\left(\frac{m\left(m^{2}+2 m-4\right)}{4} \frac{f\left(x_{n}\right)}{f^{\prime}\left(x_{n}\right)}-\frac{m(m+2)^{2}}{4}\left(\frac{m}{m+2}\right)^{m} \frac{f\left(x_{n}\right)}{f^{\prime}\left(y_{n}\right)}\right)\left[G\left(\frac{f^{\prime}\left(y_{n}\right)}{f^{\prime}\left(x_{n}\right)}\right)+H\left(\frac{f\left(x_{n}\right)}{f^{\prime}\left(x_{n}\right)}\right)\right],
\end{aligned}\right.
$$


where $G($.$) and H($.$) are two real valued weight functions.$

Moreover, in 2013 Soleymani and Babajee in [14] constructed another type of fourth-order optimal scheme, whose iterative expression is

$$
\left\{\begin{aligned}
y_{n} & =x_{n}-\frac{2 m}{m+2} \frac{f\left(x_{n}\right)}{f^{\prime}\left(x_{n}\right)}, \\
x_{n+1} & =x_{n}-\frac{4 m\left(\frac{m}{m+2}\right)^{m} f\left(x_{n}\right)}{\left(\frac{m}{m+2}\right)^{m}\left(m^{2}+2 m-4\right) f^{\prime}\left(x_{n}\right)-m^{2} f^{\prime}\left(y_{n}\right)} H\left(\frac{f^{\prime}\left(y_{n}\right)}{f^{\prime}\left(x_{n}\right)}\right),
\end{aligned}\right.
$$

being $H($.$) a real valued weight function.$

On the other hand, Zhou et al. in [17] proposed a new optimal family of fourth-order methods which requires two-function and one-derivative evaluation per iteration, that is given by

$$
\left\{\begin{array}{c}
y_{n}=x_{n}-m \frac{f\left(x_{n}\right)}{f^{\prime}\left(x_{n}\right)} \\
x_{n+1}=x_{n}-m G(v) \frac{f\left(x_{n}\right)}{f^{\prime}\left(x_{n}\right)}
\end{array}\right.
$$

where $v=\sqrt[m]{\frac{f\left(y_{n}\right)}{f\left(x_{n}\right)}}$ and $G(.) \in C^{2}(\mathbb{R})$ is a weight function. But, the problem with the application of this scheme is that when you solve for even multiplicity then you may obtain a complex root while your desired root is real number (please see Table 4 in the numerical section).

Recently, in 2015, Hueso et al. in [6] presented the following optimal fourth-order scheme for multiple roots

$$
\left\{\begin{aligned}
y_{n} & =x_{n}-\frac{2 m}{m+2} \frac{f\left(x_{n}\right)}{f^{\prime}\left(x_{n}\right)} \\
x_{n+1} & =x_{n}-\left(s_{1}+s_{2} h\left(y_{n}, x_{n}\right)+s_{3} h\left(x_{n}, y_{n}\right)+s_{4} h\left(y_{n}, x_{n}\right)^{2}\right) \frac{f\left(x_{n}\right)}{f^{\prime}\left(x_{n}\right)},
\end{aligned}\right.
$$

where $h\left(x_{n}, y_{n}\right)=\frac{f^{\prime}\left(y_{n}\right)}{f^{\prime}\left(x_{n}\right)}$ and the values of these parameters can be seen in [6].

In 2016, Behl et al. in [1] presented a new optimal scheme of fourth-order methods based on some the arithmetic mean of Chebyshev's method [10] and Schröder's method [10,12] with four free disposable parameters, which is given by

$$
\left\{\begin{aligned}
y_{n} & =x_{n}-\frac{2 m}{m+2} \frac{f\left(x_{n}\right)}{f^{\prime}\left(x_{n}\right)}, \\
x_{n+1} & =x_{n}-\frac{1}{2}\left[\frac{f\left(x_{n}\right)\left((5 m+2) b_{1} f^{\prime}\left(x_{n}\right)-(m+2) b_{2} f^{\prime}\left(y_{n}\right)\right)}{4 m\left\{f^{\prime}\left(x_{n}\right)\right\}^{2}}+\frac{2 m b_{3} f\left(x_{n}\right)}{(m-2) f^{\prime}\left(x_{n}\right) b_{4}+(m+2) f^{\prime}\left(y_{n}\right)}\right],
\end{aligned}\right.
$$

where $b_{1}, b_{2}, b_{3}$ and $b_{4}$ are free disposable parameters and the values of these parameters can be seen in [1].

One can obtain the expresssions (1), (2), (3), (6), (8) and (9) as special cases of scheme (4), just by considering suitable choice of weight functions.

It is straightforward to say from the above mentioned schemes that they have the flexibility (free parameters or weight functions) only at second step, in order to obtain new iterative methods. We want to explore the idea that what will happen if we make changes at first step as well as at the second one. Is it possible to get some faster version of optimal fourth-order methods with simple body structure, smaller asymptotic error constants and smaller residual errors when we make changes also in the first step?

Motivated and inspired by these questions, we propose a new fourth-order scheme based on weight function approach. The beauty of this manuscript is to highlight the advantages of this new approach over the earlier studies that our proposed scheme will give the flexibility to the scholars at both steps in order to construct new fourth-order iterative methods. In addition, our proposed methods not only give the faster convergence but also have smaller residual error and asymptotic error constants. Our proposed scheme only consumes three functional evaluations $\left(f\left(x_{n}\right), f^{\prime}\left(x_{n}\right)\right.$ and $\left.f^{\prime}\left(z_{n}\right)\right)$ per full iteration which satisfies the classical Kung-Traub conjecture. We have demonstrated the usefulness of the proposed methods by performing several numerical examples and observed that our methods have better numerical results, in most of the examples, than some existing methods available in the literature (in terms of smaller asymptotic error constants and smaller residual errors). Further, the dynamical study of these methods also deeps into the stability aspects of the different elements of the proposed class.

In order to study the dynamical behavior of the iterative methods included in the proposed family, it is necessary to recall some basic dynamical concepts. For a more extensive review of these concepts, see $[2,4]$. 
Let $R: \hat{\mathbb{C}} \rightarrow \hat{\mathbb{C}}$ be an operator that results from applying an iterative method on a particular function, where $\hat{\mathbb{C}}$ is the Riemann sphere. The orbit of a point $z_{0} \in \hat{\mathbb{C}}$ is defined as the set of successive images of $z_{0}$ by the operator, $\left\{z_{0}, R\left(z_{0}\right), \ldots, R^{n}\left(z_{0}\right), \ldots\right\}$.

The orbit of a point on the complex plane can be classified depending on its asymptotic behavior. In this way, a point $z_{0} \in \mathbb{C}$ is a fixed point of $R$ if $R\left(z_{0}\right)=z_{0}$. A fixed point is attracting, repelling or neutral (parabolic) if $\left|R^{\prime}\left(z_{0}\right)\right|$ is lower than, greater than or equal to 1 , respectively. Moreover, if $\left|R^{\prime}\left(z_{0}\right)\right|=0$, the fixed point is superattracting. A periodic point $z$ of period $p>1$ is a point such that $R^{p}(z)=z$ and $R^{k}(z) \neq z$, for $k<p$. A pre-periodic point is a point $z$ that is not periodic but there exists a $k>0$ such that $R^{k}(z)$ is periodic.

If $z_{f}^{*}$ is an attracting fixed point of operator $R$, its basin of attraction $\mathcal{A}\left(z_{f}^{*}\right)$ is defined as the set of pre-images of any order such that

$$
\mathcal{A}\left(z_{f}^{*}\right)=\left\{z_{0} \in \hat{\mathbb{C}}: R^{n}\left(z_{0}\right) \rightarrow z_{f}^{*}, n \rightarrow \infty\right\} .
$$

The set of points whose orbits tend to any attracting periodic point $z_{f}^{*}$ is defined as the Fatou set, $\mathcal{F}(R)$. The complementary set, the Julia set $\mathcal{J}(R)$, is the closure of the set of pre-images of its repelling fixed points, and establishes the boundaries between the basins of attraction.

The rest of the manuscript is organized in the following way: Section 2 is devoted to describe the new family of iterative methods and to study its convergence, detailing some particular cases. Some of them are used in the dynamical numerical sections. This dynamical analysis on polynomials of low degree with multiplicity 2 and 3 is made in Section 3 . Different numerical experiments to confirm the theoretical results are shown in Section 4. Finally, some conclusions and remarks are presented.

\section{Development of the optimal fourth-order scheme}

This section is devoted to the main contribution of this manuscript. Here, we will propose the optimal fourth-order iterative scheme which will gives the flexibility to the researchers at both steps in order to explore other new schemes (for the details please see the special cases). According to our knowledge, this type of problem is not discussed in the earlier studies [1,6,8, 13-15, 17, 18]. Therefore, we propose the following new fourth-order structure

$$
\begin{gathered}
y_{n}=x_{n}-h\left(x_{n}\right) \frac{f\left(x_{n}\right)}{f^{\prime}\left(x_{n}\right)}, \\
x_{n+1}=x_{n}-\frac{f\left(x_{n}\right)}{f^{\prime}\left(x_{n}\right)} G_{f}\left(u_{n}\right),
\end{gathered}
$$

where the weight function $G_{f}: \mathbb{C} \rightarrow \mathbb{C}$ is an analytic function in a neighborhood of $w=\left(\frac{m}{m+2}\right)^{m-1}, u_{n}=\frac{f^{\prime}\left(z_{n}\right)}{f^{\prime}\left(x_{n}\right)}$, $z_{n}=\frac{x_{n}+y_{n}}{2}$ and $h: \mathbb{C} \rightarrow \mathbb{C}$ is also an analytic function in the region enclosing a multiple zero $\xi$ of $f(x)$.

In the next result, we prove that the order of convergence of the proposed scheme reaches fourth-order without using any additional functional evaluation. It is interesting to observe that $h$ and $G$ have a key role in the construction of the desired fourth-order convergence.

Theorem 1 Let $x=\xi$ be a multiple zero with multiplicity $m>1$ of an analytic function $f: \mathbb{C} \rightarrow \mathbb{C}$ in the region enclosing $\xi$. Then, the family of iterative methods defined by (10) has fourth-order convergence when the following conditions hold

$$
\begin{aligned}
& h(\xi)=\frac{4 m}{m+2}, h^{\prime}(\xi)=0, \quad h^{\prime \prime}(\xi)=0, \\
& G(w)=m, \quad G^{\prime}(w)=-\frac{m^{3}}{4 p}, G^{\prime \prime}(w)=\frac{m^{4}}{4 p^{2}},
\end{aligned}
$$

where $p=\left(1-\frac{h(\xi)}{2 m}\right)^{m}$ and $\left|G^{\prime \prime \prime}(w)\right|<\infty$.

Proof Let us consider $e_{n}=x_{n}-\xi$ the error at nth iteration. Expanding in Taylor's series functions $f\left(x_{n}\right)$ and $f^{\prime}\left(x_{n}\right)$ about the point $x_{n}=\xi$ leads us:

$$
f\left(x_{n}\right)=\frac{f^{(m)}(\xi)}{m !} e_{n}^{m}\left(1+c_{1} e_{n}+c_{2} e_{n}^{2}+c_{3} e_{n}^{3}+c_{4} e_{n}^{4}+O\left(e_{n}^{5}\right)\right)
$$

and

$$
f^{\prime}\left(x_{n}\right)=\frac{f^{m}(\xi)}{m !} e_{n}^{m-1}\left(1+(m+1) c_{1} e_{n}+(m+2) c_{2} e_{n}^{2}+(m+3) c_{3} e_{n}^{3}+(m+4) c_{4} e_{n}^{4}+O\left(e_{n}^{5}\right)\right)
$$


respectively, where $c_{k}=\frac{m !}{(m+k) !} \frac{f^{(m+k)}(\xi)}{f^{(m)}(\xi)}, \quad k \geq 1$.

Now, expanding $h\left(x_{n}\right)$, we obtain

$$
h\left(x_{n}\right)=h(\xi)+e_{n} h^{\prime}(\xi)+e_{n}^{2} \frac{h^{\prime \prime}(\xi)}{2}+O\left(e_{n}^{3}\right) .
$$

From (12)- (14), we further yield

$$
\begin{aligned}
y_{n}-\xi= & \left(1-\frac{h(\xi)}{m}\right) e_{n}+\frac{h(\xi) c_{1}-m h^{\prime}(\xi)}{m^{2}} e_{n}^{2} \\
& +\frac{m\left(4 h(\xi) c_{2}-m h^{\prime \prime}(\xi)\right)-2 h(\xi) c_{1}^{2}(m+1)+2 h^{\prime}(\xi) c_{1} m}{2 m^{3}} e_{n}^{3}+O\left(e_{n}^{4}\right)
\end{aligned}
$$

Again, by using the Taylor's series expansions, we have

$$
\begin{aligned}
f^{\prime}\left(y_{n}\right) & =e_{n}^{m}\left[\frac{-2 m^{2} p}{m !(h(\xi)-2 m) e_{n}}+\frac{p\left\{c_{1}\left(h(\xi)^{2}(m+1)-2 h(\xi) m(m+3)+4 m^{2}(m+1)\right)-2 h^{\prime}(\xi)(m-1) m^{2}\right\}}{m !(h(\xi)-2 m)^{2}}\right. \\
& \left.+\frac{1}{(2 m) m !(2 m-h(\xi))^{3}} \theta_{1} e_{n}+\frac{p}{12 m^{4} m !(h(\xi)-2 m)^{2}} \theta_{2} e_{n}^{2}+O\left(e_{n}^{3}\right)\right],
\end{aligned}
$$

where

$\theta_{1}=-2 h^{\prime}(\xi) c_{1} m^{2}\left\{h(\xi)^{2}(m+1)-2 h(\xi)\left(m^{2}+4 m-1\right)+4\left(m^{3}+m\right)\right\}+2 h(\xi) c_{1}^{2} m\left\{h(\xi)^{2}(m+1)-h(\xi) m(m+7)+\right.$ $4 m(m+1)\}+c_{2}\left\{h(\xi)^{4}(m+2)-8 h(\xi)^{3} m(m+2)+8 h(\xi)^{2} m^{2}(2 m+7)-16 h(\xi) m^{3}(m+5)+16 m^{4}(m+2)\right\}+2(m-$ 1) $m^{3}\left\{h^{\prime \prime}(\xi)(h(\xi)-2 m)+h^{\prime}(\xi)^{2}(m-2)\right\}$,

$\theta_{2}=6 c_{1}(m-1)(m+1) m^{3}\left\{m\left(h^{\prime \prime}(\xi)(h(\xi)-2 m)+h^{\prime}(\xi)^{2}(m-2)\right)+2 h^{\prime}(\xi) c_{1}(h(\xi)(-m)+h(\xi)+2 m)+h(\xi) c_{1}^{2}(3 h(\xi)-4(m+\right.$ $\left.1))-4 h(\xi) c_{2}(h(\xi)-2 m)\right\}-\frac{4(m-1)(h(\xi)-2 m)}{\left(2-\frac{h(\xi)}{m}\right)^{3}}\left[3(h(\xi)-2 m)^{2}\left\{2 m^{2}\left(3 h(\xi) c_{3}+2 h^{\prime}(\xi) c_{2}\right)+c_{1} m\left(h^{\prime \prime}(\xi) m-2 h(\xi) c_{2}(3 m+4)\right)+\right.\right.$ $\left.2 h(\xi) c_{1}^{3}(m+1)^{2}-2 h^{\prime}(\xi) c_{1}^{2} m(m+1)\right\}+3(m-2)(h(\xi)-2 m)\left(h(\xi) c_{1}-h^{\prime}(\xi) m\right)\left\{m\left(h^{\prime \prime}(\xi) m-4 h(\xi) c_{2}\right)+2 h(\xi) c_{1}^{2}(m+1)-\right.$ $\left.\left.2 h^{\prime}(\xi) c_{1} m\right\}+(m-3)(m-2)\left(h(\xi) c_{1}-h^{\prime}(\xi) m\right)^{3}\right]-3 m^{5}(h(\xi)-2 m)\left(\frac{2 c_{1}(m+1)\left\{m\left(4 h(\xi) c_{2}-h^{\prime \prime}(\xi) m\right)-2 h(\xi) c_{1}^{2}(m+1)+2 h^{\prime}(\xi) c_{1} m\right\}}{m^{3}}-\right.$ $\left.\frac{4 c_{2}(m+2)(h(\xi)-2 m)\left(h(\xi) c_{1}-h^{\prime}(\xi) m\right)}{m^{3}}+c_{3}(m+3)\left(2-\frac{h(\xi)}{m}\right)^{3}\right)+6(m-1) m^{2}\left(h(\xi) c_{1}-h^{\prime}(\xi) m\right)\left\{c_{2}(m+2)(h(\xi)-2 m)^{2}+\right.$ $\left.2 h(\xi) c_{1}^{2}(m+1)-2 h^{\prime}(\xi) c_{1} m(m+1)\right\}$

With the help of expressions (13) and (17), we get

$$
\begin{aligned}
u_{n}= & \frac{f^{\prime}\left(z_{n}\right)}{f^{\prime}\left(x_{n}\right)}=-\frac{2 m p}{h(\xi)-2 m}+\frac{p\left[c_{1} h(\xi)\{h(\xi)(m+1)-4 m\}-2 h(\xi)^{\prime}(\xi)(m-1) m^{2}\right]}{m(h(\xi)-2 m)^{2}} e_{n} \\
& -\frac{p}{2 m^{2}(h(\xi)-2 m)^{3}}\left[2 c_{1}^{2} h(\xi)\left\{h(\xi)^{2}\left(2 m^{2}+3 m+1\right)-3 h(\xi) m\left(m^{2}+5 m+2\right)+12 m^{2}(m+1)\right\}\right. \\
& -2 c_{1} h^{\prime}(\xi) m^{2}\left\{h(\xi)^{2}(m+1)-8 m(h(\xi)-1)\right\}+c_{2} h(\xi)\left\{h(\xi)^{3}(m+2)-8 h(\xi)^{2} m(m+2)-48 m^{3}\right. \\
& \left.\left.+12 h(\xi) m^{2}(m+4)\right\}+2(m-1) m^{3}\left\{h^{\prime \prime}(\xi)(h(\xi)-2 m)+h^{\prime}(\xi)^{2}(m-2)\right\}\right] e_{n}^{2}+O\left(e_{n}^{3}\right) .
\end{aligned}
$$

It is clear from expression (18) that $u_{n}=w+O\left(e_{n}\right)$, where $w=-\frac{2 m p}{h(\xi)-2 m}$. Therefore, we expand the weight function $G_{f}\left(u_{n}\right)$ in the neighborhood of $w$ :

$$
G_{f}\left(u_{n}\right)=G_{f}(w)+G_{f}^{\prime}(w) u_{n}+\frac{1}{2 !} G_{F}^{\prime \prime}(w) u_{n}^{2}+\frac{1}{3 !} G_{f}^{\prime \prime \prime}(w) u_{n}^{3}+O\left(u_{n}^{4}\right) .
$$

By using expressions (12)-(19), in the last step of the proposed scheme, we obtain

$$
e_{n+1}=\left(1-\frac{G(w)}{m}\right) e_{n}+\sum_{j=2}^{4} M_{j} e_{n}^{j}+O\left(e_{n}^{5}\right)
$$

where $M_{j}=M_{j}\left(h(\xi), h^{\prime}(\xi), h^{\prime \prime}(\xi), m, c_{1}, c_{2}, c_{3}, c_{4}\right), j=2,3,4$.

From expression (20), our scheme (10) reaches at least second-order of convergence if

$$
G_{f}(w)=m .
$$


By using the above value of $G_{f}(w)$ in $M_{2}=0$, we obtain the following two independent relations

$$
\begin{aligned}
m-\frac{G_{f}^{\prime}(w) h(\xi) p\{h(\xi)(m+1)-4 m\}}{(h(\xi)-2 m)^{2}} & =0, \\
\frac{2 G_{f}^{\prime}(w) h^{\prime}(\xi)(m-1) m^{2} p}{(h(\xi)-2 m)^{2}} & =0,
\end{aligned}
$$

which further yield

$$
h^{\prime}(\xi)=0, \quad G_{f}^{\prime}(w)=\frac{m(h(\xi)-2 m)^{2}}{p h(\xi)(h(\xi) m+h(\xi)-4 m)} .
$$

In order to obtain an optimal fourth-order convergence, we will use the above expressions (21) and (22) in $M_{3}=0$. Then, we have after some simplifications

$$
h(\xi)=\frac{4 m}{m+2}, \quad h^{\prime \prime}(\xi)=0, \quad G_{f}^{\prime \prime}(w)=\frac{1}{4 p^{2}} m^{4}
$$

Finally, by using expressions (21), (22) and (23) in (20) in order to obtain asymptotic error constant, we get

$$
e_{n+1}=\frac{(m+2)^{2}\left(32 p^{3} G_{f}^{\prime \prime \prime}(w)+m^{9}+2 m^{8}+2 m^{7}-2 m^{6}+12 m^{5}\right) c_{1}^{3}+3 m^{11} c_{3}-3 m^{9}(m+2)^{2} c_{1} c_{2}}{3 m^{10}(m+2)^{2}} e_{n}^{4}+O\left(e_{n}^{5}\right)
$$

where $\left|G^{\prime \prime \prime}(w)\right|<+\infty$.

The above expression (24) demonstrate that our proposed scheme (10) reaches fourth-order convergence by consuming only three functional evaluations per iteration. Hence, the scheme (10) have also reached the optimal order of convergence in the sense of Kung-Traub conjecture. This completes the proof.

\subsection{Some special cases}

In this section, we will discuss some special cases of our proposed scheme (10) by assigning different $h(x)$ and weight function $G_{f}$. In this regard, please see Table 1 where we have mentioned some different kind of members of the family. In addition, we choose arbitrary any $h(x)$ and $G(x)$ from the table in order to obtain new optimal methods of fourth-order.

\begin{tabular}{|c|c|l|}
\hline Cases & $h(x)$ & $G_{f}(u)$ \\
\hline Case-1 & $\frac{4 m}{m+2}$ & $m-\frac{m^{3}}{4 p}(u-w)+\frac{m^{4}}{4 p^{2}}(u-w)^{2}+G_{3}(u-w)^{3}, G_{3}=G^{\prime \prime \prime}(w)$. \\
\hline Case-2 & $\frac{4 m}{m+2}$ & $-\frac{m(m(p-u)+2 u)}{2(p-u)}$ \\
\hline Case-3 & $\frac{4 m}{m+2}$ & $\begin{array}{l}\frac{a_{2}}{u^{2}}+\frac{1}{8} \frac{m\left((2+m)^{4} p^{2}-24 a_{2}\right)}{(2+m) p u}+\frac{1}{8} \frac{m^{3}\left(m(2+m)^{3} p^{2}-8 a_{2}\right) u}{(2+m)^{3} p^{3}} \\
+\frac{3 a_{2} m^{2}}{(2+m)^{2} p^{2}}-\frac{m}{4}\left(m^{3}+3 m^{2}+2 m-4\right), \text { where } a_{2} \in \mathbb{R} .\end{array}$ \\
\hline Case-4 & $\frac{4 m}{m+2}+f\left(x_{n}\right)^{m}$ & $\begin{array}{l}\frac{a_{2}}{u^{2}}+\frac{1}{8} \frac{m\left((2+m)^{4} p^{2}-24 a_{2}\right)}{(2+m) p u}+\frac{1}{8} \frac{m^{3}\left(m(2+m)^{3} p^{2}-8 a_{2}\right) u}{(2+m)^{3} p^{3}} \\
+\frac{3 a_{2} m^{2}}{(2+m)^{2} p^{2}}-\frac{m}{4}\left(m^{3}+3 m^{2}+2 m-4\right), \text { where } a_{2} \in \mathbb{R} .\end{array}$ \\
\hline Case-5 & $\frac{4 m}{m+2} \cos \left(f\left(x_{n}\right)\right)$ & $-\frac{m(m(p-u)+2 u)}{2(p-u)}$ \\
\hline Case-6 & $\frac{4 m}{m+2} e^{\sin \left(f\left(x_{n}\right)\right)}$ & $m-\frac{m^{3}(u-w)+\frac{m^{4}}{4 p}(u-w)^{2}+G_{3}(u-w)^{3}, G_{3}=G^{\prime \prime \prime}(w)}{2(p-u)}$ \\
\hline Case-7 & $\frac{4 m}{m+2} a^{f\left(x_{n}\right)}, a>0$ & $-\frac{m(m(p-u)+2 u)}{2(p-u)}$ \\
\hline
\end{tabular}

Table 1: Some special cases of the the proposed scheme (10).

Let us remark that, observing the error equation $24, G_{3}=G^{\prime \prime \prime}(w)=0$ and $G_{3}=-\frac{m^{5}}{32 p^{3}}\left(m^{4}+2 m^{3}+2 m^{2}-2 m+12\right)$ reduce the expression of the asymptotic constant error. Case- 1 is used in the dynamical analysis described in the next section and cases 1 to 4 are used in the numerical experiments.

As the order of the methods does not depend on the value of $G_{3}$, this element can be considered as a free parameter in order to analyze the stability of the resulting class. This study will reveal which can be the better complex value to be assigned to $G_{3}$ and how the behavior of the elements of the class changes with increasing multiplicity. 


\section{Dynamical analysis in the complex plane}

Blanchard in [2] considered the conjugacy map (a Möbius transformation)

$$
k(z)=\frac{z-a}{z-b}
$$

with the following properties:

$$
\text { i) } k(\infty)=1, \text { ii) } k(a)=0, \quad(i i i) k(b)=\infty,
$$

and proved that, for quadratic polynomials, Newton's operator is conjugate to the rational map $z^{2}$. We use this map in order to avoid the appearance of parameters $a$ and $b$ in the rational functions resulting from applying the fixed point operator of the iterative method on a polynomial $p(z)$ with roots $a$ and $b$.

\subsection{Study on a polynomial with double roots}

In order to study the stability of the family on polynomials with double roots, the operator of the family on $p(z)=(z-$ $a)^{m}(z-b)$ with $m=2$ is calculated, obtaining a rational function that depends, not only on the parameter $G_{3}$, but also on parameters $a$ and $b$. In order to eliminate these parameters, the following transformation is usually applied.

Next we are going to analyze, under the dynamical point of view, the stability and reliability of the members of the proposed family on $p(z)$. Firstly, we will study the fixed points of the rational function that are not related with the original roots of the polynomial and the free critical points, that is, the critical points of the associated rational function different from 0 and $\infty$.

For $p(z)$, the operator associated to family (10) for Case-1 (see Table 1) is the rational function $M_{p}\left(z, G_{3}, a, b\right)$ depending on the parameters $G_{3}, a$ and $b$. On the other hand, operator $M_{p}\left(z, G_{3}, a, b\right)$ on $p(z)$ is conjugated to

$$
O_{p}\left(z, G_{3}\right)=\left(k \circ M_{p} \circ k^{-1}\right)(z)=z^{4} \frac{-G_{3}\left(-2-2 z+z^{2}\right)^{3}+48(2+z)^{3} s_{1}(z)}{s_{2}(z)+s_{3}\left(z, G_{3}\right)}
$$

where $s_{1}(z)=64+152 z+108 z^{2}+8 z^{3}+z^{4}, s_{2}(z)=49152+196608 z+307200 z^{2}+184320 z^{3}$ and $s_{3}\left(z, G_{3}\right)=8(-8448+$ $\left.G_{3}\right) z^{4}+24\left(-6784+G_{3}\right) z^{5}+12\left(-6528+G_{3}\right) z^{6}-16\left(192+G_{3}\right) z^{7}-6\left(-992+G_{3}\right) z^{8}+6\left(64+G_{3}\right) z^{9}-\left(240+G_{3}\right) z^{10}$. Let us remark that, by means of this transformation, the original roots $a$ and $b$ have been obviated in $O_{p}\left(z, G_{3}\right)$, becoming 0 and $\infty$.

However, it is possible to reduce this rational function to simpler one, for specific values of the parameter $G_{3}$, as it is presented in the following result.

Proposition 1 The degree of the rational function $O_{p}\left(z, G_{3}\right)$ associated to family $(10)$ on $p(z)$ can be reduced in the following cases:

- If $G_{3}=0$, the rational operator is

$$
O_{p}(z, 0)=-z^{4} \frac{64+152 z+108 z^{2}+8 z^{3}+z^{4}}{-128-320 z-224 z^{2}+112 z^{3}+216 z^{4}+44 z^{5}-38 z^{6}+5 z^{7}}
$$

- For $G_{3}=-15984$, the fixed point operator is reduced to

$$
O_{p}(z,-15984)=z^{4} \frac{2152+8160 z+9084 z^{2}+1420 z^{3}-1482 z^{4}+348 z^{5}+z^{6}}{2\left(-512-2560 z-5760 z^{2}-7680 z^{3}-5644 z^{4}+48 z^{5}+2862 z^{6}+230 z^{7}-831 z^{8}+164 z^{9}\right)}
$$

Some of these values of $G_{3}$ will appear again when we analyze the lowest number of strange fixed and critical points of the rational function $O_{p}\left(z, G_{3}\right)$. 


\subsubsection{Fixed and critical points}

Once we have obtained a rational function $O_{p}\left(z, G_{3}\right)$ depending only of the free parameter $G_{3}$, we must find its associated fixed and critical points in order to analyze their asymptotic behavior. Strange fixed points are obtained from solving equation $O_{p}\left(z, G_{3}\right)=z$, that is,

$$
z(z-1) r(z)=0
$$

where

$$
\begin{aligned}
r(z)= & 49152+245760 z+552960 z^{2}+\left(712704-8 G_{3}\right) z^{3}+\left(549888-24 G_{3}\right) z^{4}+\left(239616-12 G_{3}\right) z^{5} \\
& \left(49152+16 G_{3}\right) z^{6}+\left(2688+6 G_{3}\right) z^{7}+\left(576-6 G_{3}\right) z^{8}+\left(288+G_{3}\right) z^{9} .
\end{aligned}
$$

It is clear that $O_{p}\left(z, G_{3}\right)$ has 0 and $\infty$ as fixed points (corresponding to the multiple and simple roots $a$ and $b$, respectively), but also different fixed points that do not correspond to the roots of $p(z): z=1$ and the roots of $r(z)$ that will be denoted by $r_{i}\left(G_{3}\right), i=1, \ldots, 9$ and are called strange fixed points. However, the complexity of the operator can be lower depending on the value of the parameter, as we can see in the following result. By replacing parameter $G_{3}$ by different values and after some algebraic manipulations, we obtain the following result.

Theorem 2 The number of strange fixed points of operator $O_{p}\left(z, G_{3}\right)$ is ten (including $z=1$, for $G_{3} \neq-15984$ ), except in the following cases:

(i) For $G_{3}=-15984, z=1$ is not a strange fixed point and the rational operator was presented in Proposition 1 . So, the strange fixed points are the specific roots of $r(z)$ for this value of the parameter.

(ii) For $G_{3}=88992, z=1$ is a strange fixed point with multiplicity 3 and also there exist seven strange fixed points more of the rational function

$$
O_{p}(z, 88992)=z^{4} \frac{-1854\left(-2-2 z+z^{2}\right)^{3}+(2+z)^{3}\left(64+152 z+108 z^{2}+8 z^{3}+z^{4}\right)}{q_{1}(z)},
$$

where $q_{1}(z)=1024+4096 z+6400 z^{2}+3840 z^{3}+13424 z^{4}+41104 z^{5}+20616 z^{6}-29728 z^{7}-11000 z^{8}+11132 z^{9}-$ $1859 z^{10}$.

(iii) For $G_{3}=0$, there are seven strange fixed points, $z=1$ and the roots of polynomial $64+224 z+336 z^{2}+248 z^{3}+64 z^{4}-$ $12 z^{5}+3 z^{6}$, being the rational operator

$$
O_{p}(z, 0)=-z^{4} \frac{64+152 z+108 z^{2}+8 z^{3}+z^{4}}{-128-320 z-224 z^{2}+112 z^{3}+216 z^{4}+44 z^{5}-38 z^{6}+5 z^{7}} .
$$

(iv) For $G_{3}=-57888$, there are nine strange fixed points: $z=-\frac{1}{2}, z=1, z=4$ and the roots of $64+80 z+228 z^{2}+$ $500 z^{3}+46 z^{4}-228 z^{5}+75 z^{6}$, being the associated rational function

$$
O_{p}(z,-57888)=z^{4} \frac{-9136-26960 z-11400 z^{2}+21632 z^{3}+8140 z^{4}-7068 z^{5}+1220 z^{6}+z^{7}}{q_{2}(z)}
$$

where $q_{2}(z)=1024+4096 z+6400 z^{2}+3840 z^{3}-11056 z^{4}-32336 z^{5}-16104 z^{6}+19232 z^{7}+7360 z^{8}-7228 z^{9}+1201 z^{10}$.

As important as the number of strange fixed point is their asymptotic behavior, as in the most of cases their stability depend on the parameter of the family. The expression of the differential operator, needed to analyze this stability and to obtain the critical points, is

$$
O_{p}^{\prime}\left(z, G_{3}\right)=48 z^{3}(2+z)^{9} \frac{G_{3}\left(-2-2 z+z^{2}\right)^{2} q_{3}(z)+48(2+z)^{3} q_{4}(z)}{\left(49152+196608 z+307200 z^{2}+184320 z^{3}+q_{5}\left(z, G_{3}\right)\right)^{2}},
$$

where $q_{3}(z)=-16-4 z+36 z^{2}-26 z^{3}+z^{4}, q_{4}(z)=-512-944 z-32 z^{2}+620 z^{3}-136 z^{4}+5 z^{5}$ and $q_{5}\left(z, G_{3}\right)=8(-8448+$ $\left.G_{3}\right) z^{4}+24\left(-6784+G_{3}\right) z^{5}+12\left(-6528+G_{3}\right) z^{6}-16\left(192+G_{3}\right) z^{7}-6\left(-992+G_{3}\right) z^{8}+6\left(64+G_{3}\right) z^{9}-\left(240+G_{3}\right) z^{10}$.

By definition, it is clear that $z=0$ is always a superattracting fixed point, as it comes from the double root of the polynomial. However, the stability of $z=\infty$ and the rest of fixed points depends on the value of parameter $G_{3}$. In the following results we establish the stability of these fixed points.

Theorem 3 The character of $z=\infty$ depends on $G_{3}$ as follows:

(i) If $\left|240+G_{3}\right|<48$, then $z=\infty$ is an attractor, being superattractive if $G_{3}=-240$. 
(ii) When $\left|240+G_{3}\right|=48, z=\infty$ is a parabolic point.

(iii) If $\left|240+G_{3}\right|>48$, then $z=\infty$ is repulsive.

Proof In order to analyze the stability of $z=\infty$, its inverse relationship with $z=0$ (induced by Möbius transformation) is used to define the associate rational function:

$$
\operatorname{Inf}\left(z, G_{3}\right)=\frac{1}{O_{p}\left(1 / z, G_{3}\right)}=z \frac{G_{3}\left(-1+2 z+2 z^{2}\right)^{3}+48(1+2 z)^{3} q_{6}(z)}{48-\left(\left(-672+G_{3}\right) z+q_{7}\left(z, G_{3}\right)\right)},
$$

where $q_{6}(z)=-5+38 z-44 z^{2}-216 z^{3}-112 z^{4}+224 z^{5}+320 z^{6}+128 z^{7}$ and $q_{7}\left(z, G_{3}\right)=6\left(1344+G_{3}\right) z^{2}-6(-7232+$ $\left.G_{3}\right) z^{3}-16\left(-7008+G_{3}\right) z^{4}+12\left(12288+G_{3}\right) z^{5}+24\left(3968+G_{3}\right) z^{6}+8\left(3072+G_{3}\right) z^{7}$. The rest of the proof is straightforward as the stability function of the infinity is $\left|\operatorname{In} f^{\prime}\left(0, G_{3}\right)\right|=\left|\frac{240+G_{3}}{48}\right|$.

In Figure 1, the stability function of the infinity (as a fixed point) can be observed. It is worthy to notice that, all the methods of the class defined by means of values of $G_{3}$ out of the region of the complex plane where $\infty$ is attracting (the cone presented in the figure), are not suitable to find the simple root of the original polynomial $p(z)$, as it is a repulsive fixed point. However, for values of $G_{3}$ inside this stable area, both roots, simple and multiple, can be found with quartic and quadratic order of convergence, respectively.

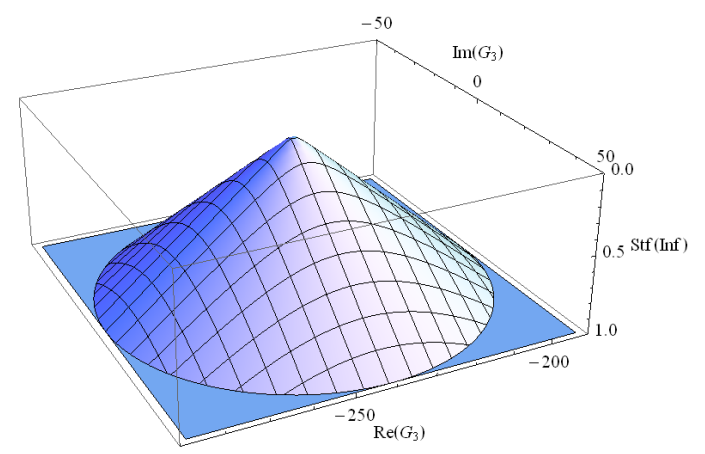

Fig. 1: Stability function of the infinity $\left|\operatorname{Inf}^{\prime}\left(0, G_{3}\right)\right|$

Theorem 4 The character of the strange fixed point $z=1$ is as follows (except for $G_{3}=-15984$ ):

(i) If $\left|G_{3}+15984\right|>104976$, then $z=1$ is an attractor and it cannot be a superattractor.

(ii) When $\left|G_{3}+15984\right|=104976, z=1$ is a parabolic point.

(iii) If $\left|G_{3}+15984\right|<104976$, then $z=1$ is a repulsor.

Proof It can be easily seen that

$$
O_{p}^{\prime}\left(1, G_{3}\right)=\frac{104976}{15984+G_{3}}
$$

So,

$$
\left|\frac{104976}{15984+G_{3}}\right| \leq 1 \text { is equivalent to } 104976 \leq\left|15984+G_{3}\right| .
$$

Let us consider $G_{3}=a_{1}+i a_{2}$ an arbitrary complex number. Then,

$$
\left(a_{1}+15984\right)^{2}+a_{2}^{2} \geq 104976^{2} .
$$

Therefore,

$$
\left|O_{p}^{\prime}\left(1, G_{3}\right)\right| \leq 1 \text { iff }\left|G_{3}+15984\right| \geq 104976 .
$$

Taking into account that $G_{3} \neq 15984$, for which $z=1$ is not a fixed point, it will be repulsive if $G_{3}$ satisfies $\left|G_{3}+15984\right|<$ 104976. 


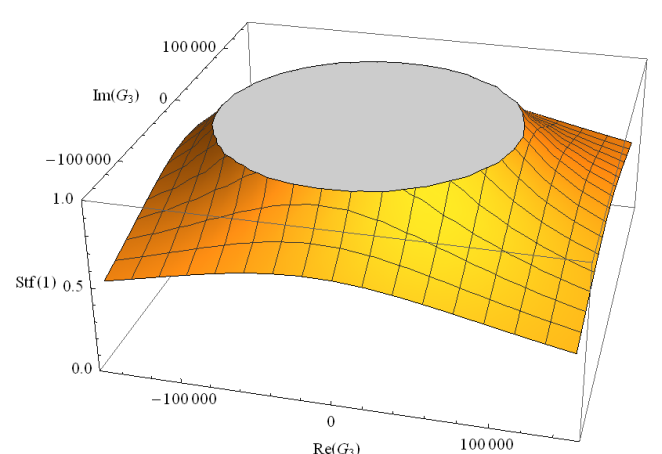

(a) $\left|O_{p}^{\prime}\left(1, G_{3}\right)\right|$

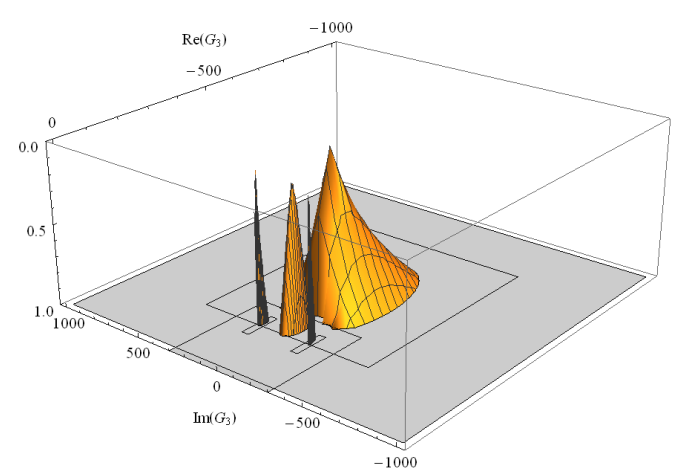

(c) $\left|O_{p}^{\prime}\left(r_{i}\left(G_{3}\right), G_{3}\right)\right|, i=1,4,7,9$

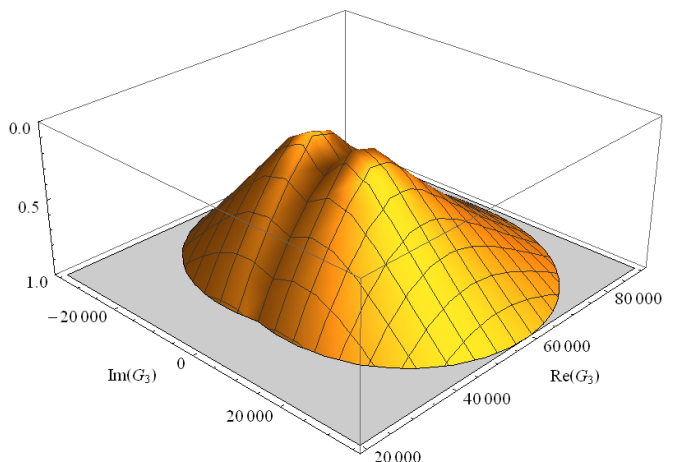

(b) $\left|O_{p}^{\prime}\left(r_{7}\left(G_{3}\right), G_{3}\right)\right|$

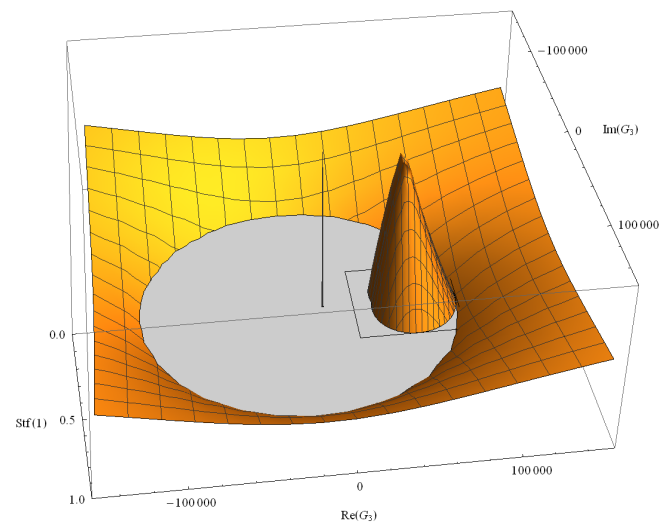

(d) Stability area

Fig. 2: Stability functions of the infinity and all the strange fixed points

The stability region corresponding to the strange fixed point $z=1$ can be seen at Figure $2 \mathrm{a}$. As it is repulsive inside the disk $\left|G_{3}+15984\right|=104976$, and $z=1$ comes from the divergence in the original performance of the problem, it can be concluded that inside this disk, the methods of the family will converge. Now, the problem is to avoid pathological convergence, as due to strange attracting fixed points or periodic orbits.

The analysis of the stability function of the rest of strange fixed points, $\left|O_{p}^{\prime}\left(r_{i}\left(G_{3}\right), G_{3}\right)\right|$, where $i=1,2, \ldots, 9$ gives us relevant information about their stability and, therefore, on the whole stability of the iterative methods of the class. In these cases, the analytical expression of the strange fixed points is not available (as they are known as roots of a certain high-degree polynomial) and, then, they are studied graphically. In Figures $2 \mathrm{~b}$ and $2 \mathrm{c}$, the stability functions of $r_{i}\left(G_{3}\right)$, for $i=1,4,7,9$, are plotted, presenting a big area in the region $[20000,90000] \times[-30000,30000]$ and a smaller one at $[-600,-200] \times[-200,200]$ of the complex plane where some of them are attracting. However, out of this region and inside the much bigger area of repulsion of $z=1$ (see Figure $2 \mathrm{~d}$ for a global view), only the roots are attracting fixed points.

Regarding the critical points, they are calculated by solving $O_{p}^{\prime}\left(z, G_{3}\right)=0$ and are presented in the following result. The relevance of the knowledge of the free critical points is the following known fact: each invariant Fatou component holds, at least, one critical point. So, the lower is the number of this kind of points, the lower number of basins of attraction is possible.

Theorem 5 The free critical points of operator $O_{p}\left(z, G_{3}\right)$ are $z=-2$, that is a pre-image of the strange fixed point $z=1$, and the roots of polynomial

$$
\begin{aligned}
c(t)= & -196608-64 G_{3}+\left(-657408-144 G_{3}\right) t+\left(-703488+112 G_{3}\right) t^{2}+\left(-76800+248 G_{3}\right) t^{3} \\
& +\left(250368-204 G_{3}\right) t^{4}+\left(100608-140 G_{3}\right) t^{5}+\left(-6528+140 G_{3}\right) t^{6}+\left(-5088-30 G_{3}\right) t^{7}+\left(240+G_{3}\right) t^{8},
\end{aligned}
$$

denoted by $c_{i}\left(G_{3}\right), i=1,2, \ldots, 8$. So, the number of free critical points (including the pre-periodic one) is nine, except in the following cases:

(i) For $G_{3}=0$, the free critical points are $z=-m=-2$ and the roots of polynomial $-512-944 t-32 t^{2}+620 t^{3}-136 t^{4}+$ $5 t^{5}$, being two of them complex conjugate.

(ii) For $G_{3}=-3072, z=-2$ and the roots of $4480+21824 t+17472 t^{2}-18272 t^{3}-11056 t^{4}+9096 t^{5}-1814 t^{6}+59 t^{7}$ are the free critical points (four of them are conjugated). 
(iii) For $G_{3} \approx-2777.8$, there are eight free critical points (including $z=-2$ ), four of them complex.

(iv) For $G_{3}=-15984$, there are three real free critical points, $z=-2$ and two roots of the polynomial $-4304-17172 t-$ $17052 t^{2}+4114 t^{3}+6993 t^{4}-2307 t^{5}+82 t^{6}$, being the rest of them complex.

(v) If $G_{3} \approx-282.7$, there exist eight free critical points, four of them real.

(vi) If $G_{3} \approx 14243.7$, there are eight free critical points, two of them complex.

As we have seen, the number of free critical points is very high and only $z=-2$ is dependent (as pre-image of $z=1$ it can be only an image of the stability of $z=1$ ) and can be avoided in the analysis. So, the parameter space is defined in $\mathbb{C}^{8}$, and it is not possible to analyze it carefully. However, some projections can be plotted, using each one of these critical points as starting guesses for the family of iterative methods. By using the routines appearing in [3], we make a mesh of $400 \times 400$ points for the values of parameter $G_{3}$ and, for each of these values, the corresponding method is applied on polynomial $p(z)$ with the critical point as initial estimation. At the end, if the method has converged to zero or has a modulus greater than $10^{6}$ in a maximum of 200 iterations, the point of the mesh is painted in red; if not, it is painted in black. In Figure 3 we can see some of these parameter planes, corresponding to different critical points.

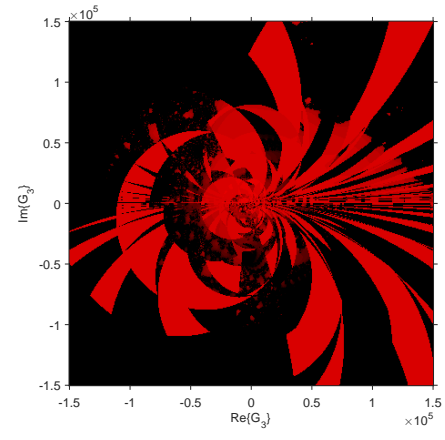

(a) $c_{1}\left(G_{3}\right)$

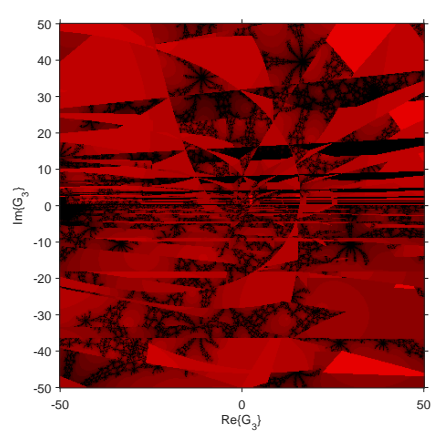

(b) $c_{1}\left(G_{3}\right)$, a detail

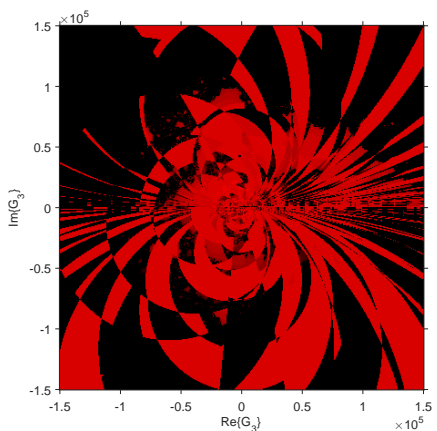

(c) $c_{2}\left(G_{3}\right)$

Fig. 3: Some parameter planes by using $c_{i}\left(G_{3}\right), i=1,2$ as initial estimations

In Figure 3a, the disk corresponding to the stability function of $z=1$ can be observed as a stable region to choose values of the parameter. Moreover, many areas of alternate stable and unstable behavior seem to be generated in a region close to the origin (the same happens in case of $c_{2}\left(G_{3}\right)$ used as initial estimation see Figure 3c)). Specifically, Figure $3 \mathrm{~b}$ is a detail of the parameter plane where some black regions appear close to the origin. As we have seen in the analysis of the stability of strange fixed points that they are all repulsive in this area, these black regions must correspond to values of $G_{3}$ with attracting periodic orbits, as will be confirmed in the following section.

\subsubsection{Dynamical Planes}

In this section we will show, by means of dynamical planes, the qualitative behavior of the different elements of the proposed family by using the conclusions obtained in the analysis of the stability of strange fixed points.

These dynamical planes has been generated by means of the routines appearing in [3]. The dynamical plane associated to a value of the parameter $G_{3}$, that is, obtained by iterating an element of family, is generated by using each point of the complex plane as initial estimation (we have used a mesh of $800 \times 800$ points). We paint in blue the points whose orbits converge to infinity (absolute value greater than $0.8 \cdot 10^{6}$ ), in orange the points converging to zero (with a tolerance of $10^{-3}$ ), in green, red, etc. those points whose orbits converge to one of the strange fixed points and in black if it reaches the maximum number of 200 iterations without converging to any of the fixed points. The color is brighter for lower number of iterations needed to converge. Moreover, each fixed point appears marked as a white circle in the figures, with a white star if the fixed point is an attractor and with a white square if it is critical.

Some values of the parameter whose associate iterative method shows stable behavior with convergence only to the "roots" (multiple $z=0$ or simple $z=\infty$ ) are presented in Figure 4. Depending on the value of the parameter, only the basin of attraction of $z=0$ is observed (global convergence to the multiple root, as is shown in Figures $4 \mathrm{c}$ to $4 \mathrm{f}$ ), or both $z=0$ and $z=\infty$ appear (Figures $4 \mathrm{a}$ and $4 \mathrm{~b}$ ). 


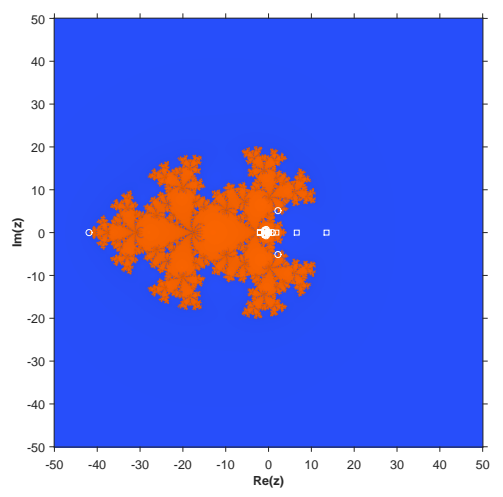

(a) $G_{3}=-240$

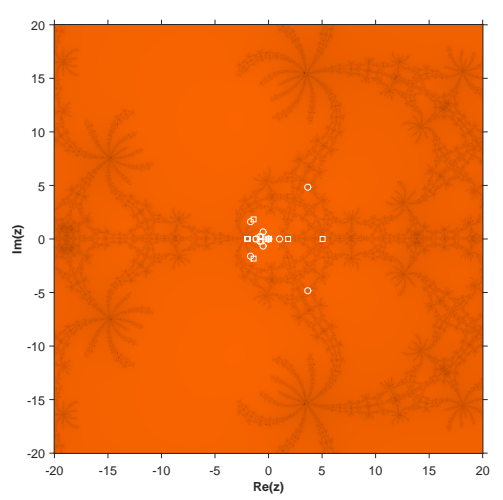

(d) $G_{3}=100$

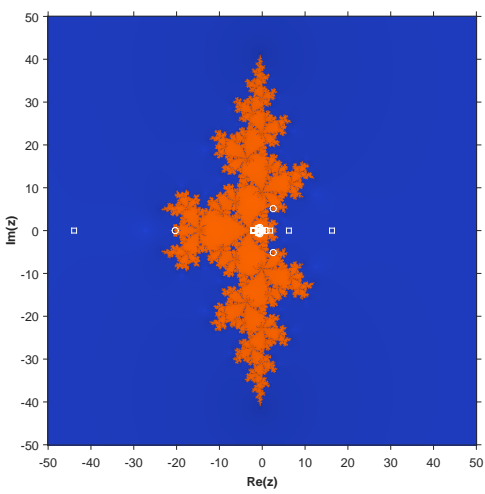

(b) $G_{3}=-200$

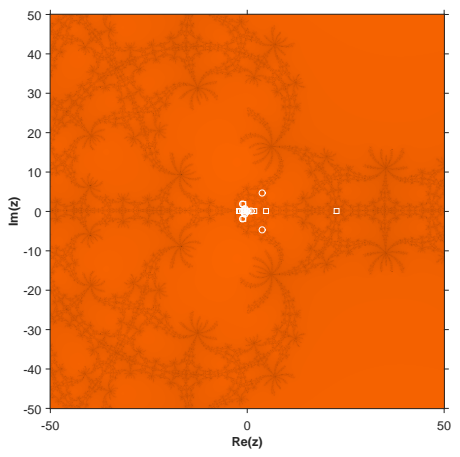

(e) $G_{3}=200$

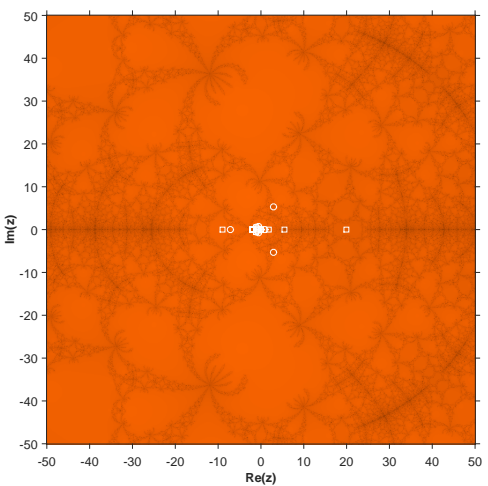

(c) $G_{3}=-100$

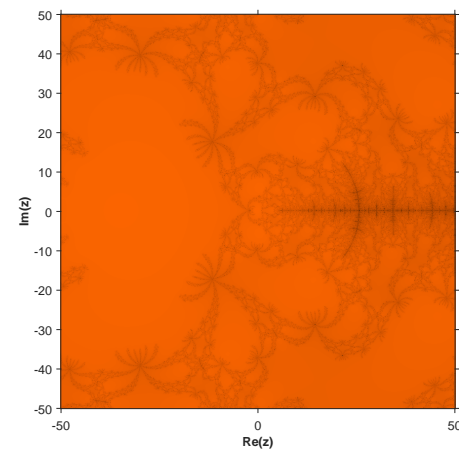

(f) $G_{3}=1000$

Fig. 4: Dynamical planes with stable behavior for $m=2$

On the other hand, unstable behavior is found when we choose values of the parameter in the stability region of attracting strange fixed points: in Figure 5a, $z=17.65$ is an attracting strange fixed point (whose basin of attraction appears in green color), a similar case is presented in Figure 5b, where $z=0.92 \pm 0.58 i$ are simultaneously attracting, or in Figure 5c, where $z=1$ has an immense basin of attraction. On the other hand, some attracting periodic orbits appear, as in Figure 5d, where the real orbit $\{5.10,-42.75,6.29,-36.17\}$ can be observed. Also in Figures $5 \mathrm{e}$ and $5 \mathrm{f}$, the dynamical plane corresponding to $G_{3}=10$ shows in black the basin of attraction of the periodic orbit $\{5.26,-42.36,5.99,-38.15\}$. The periodic orbits are marked with yellow lines, with yellow circles at the elements of the orbit.

\subsection{Complex dynamics on cubic roots}

For $q(z)=(z-a)^{3}(z-b)$, the operator associated to family (10) is the rational function $M_{q}\left(z, G_{3}, a, b\right)$ depending on parameters $G_{3}, a$ and $b$. On the other hand, by means of Möbius transformation, operator $M_{q}\left(z, G_{3}, a, b\right)$ on $q(z)$ is conjugated to

$$
O_{q}\left(z, G_{3}\right)=\left(h \circ M_{q} \circ h^{-1}\right)(z)=z^{4} \frac{-96 G_{3}\left(-81-90 z+15 z^{2}+20 z^{3}\right)^{3}+1953125(3+z)^{4} u_{1}(z)}{u_{2}(z)+u_{3}\left(z, G_{3}\right)},
$$

where $u_{1}(z)=38637+87804 z+79191 z^{2}+30888 z^{3}+3363 z^{4}-404 z^{5}+9 z^{6}, u_{2}(z)=28025208984375+93417363281250 z+$ $121442572265625 z^{2}+53974476562500 z^{3}$ and $u_{3}\left(z, G_{3}\right)=472392\left(-91796875+108 G_{3}\right) z^{4}+65610(-1172265625+$ $\left.2592 G_{3}\right) z^{5}+65610\left(-721484375+2448 G_{3}\right) z^{6}-29160\left(419140625+1056 G_{3}\right) z^{7}-91125\left(-11421875+1248 G_{3}\right) z^{8}-$ $12150\left(-119453125+2208 G_{3}\right) z^{9}+2025\left(131953125+12128 G_{3}\right) z^{10}+4500\left(-5546875+2016 G_{3}\right) z^{11}-6750(2015625+$ $\left.256 G_{3}\right) z^{12}-250\left(4984375+3072 G_{3}\right) z^{13}$. Let us observe that again, parameters $a$ and $b$ have been obviated in the rational function resulting from the Möbius transformation. Indeed, this rational function is highly simplified for $G_{3}=0$, as

$$
O_{q}(z, 0)=z^{4} \frac{38637+87804 z+79191 z^{2}+30888 z^{3}+3363 z^{4}-404 z^{5}+9 z^{6}}{177147+354294 z+177147 z^{2}-157464 z^{3}-236925 z^{4}-95904 z^{5}+7749 z^{6}+13392 z^{7}+690 z^{8}-638 z^{9}} .
$$




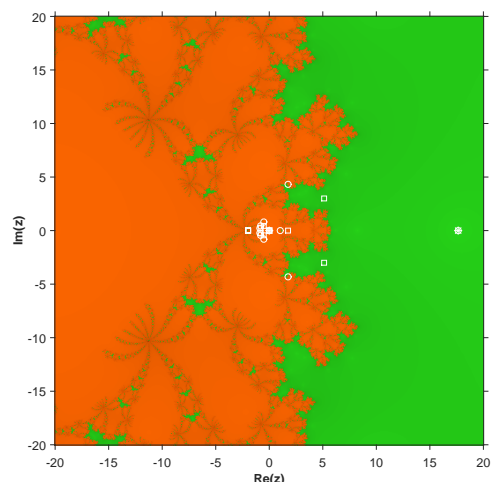

(a) $G_{3}=-500$

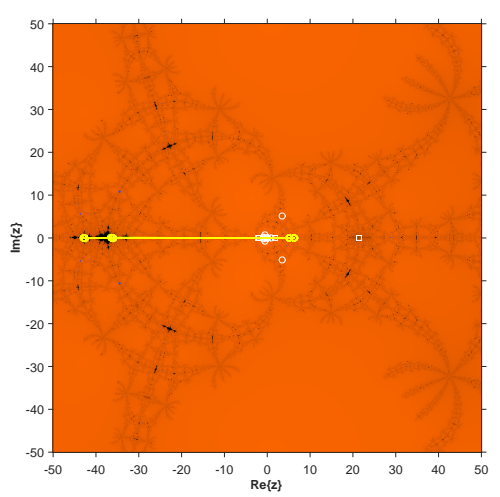

(d) $G_{3}=0$ periodic orbit

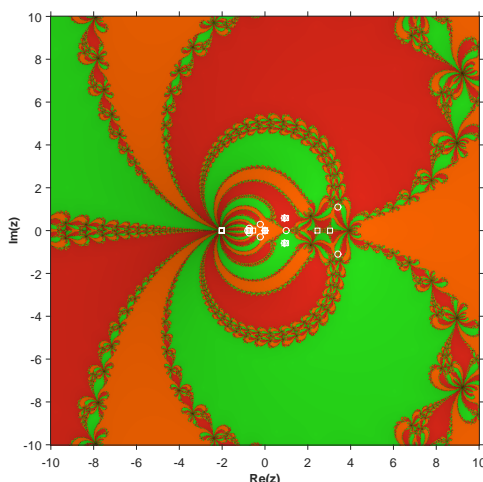

(b) $G_{3}=50000$

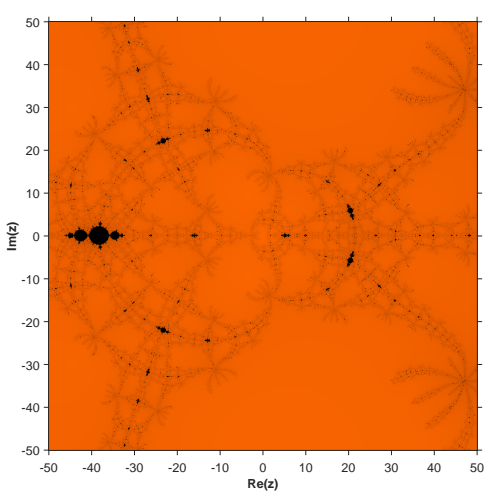

(e) $G_{3}=10$

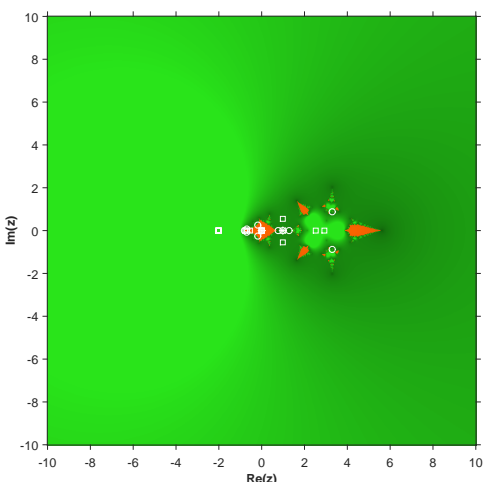

(c) $G_{3}=100000$

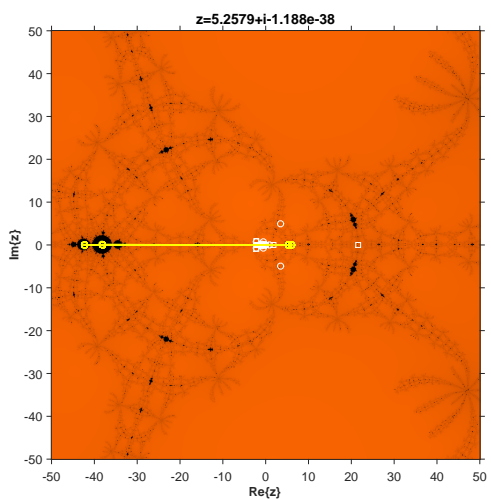

(f) $G_{3}=10$ periodic orbit

Fig. 5: Dynamical planes with unstable behavior for $m=2$

\subsubsection{Fixed and critical points}

As we have seen, the proposed fourth-order family of iterative methods, applied on the polynomial $q(z)$, and after Möbius transformation, gives rise to the rational function (27), depending on parameter $G_{3}$. It is clear that this rational function has 0 and $\infty$ as fixed points, but also different strange fixed points (obtained in the same way as in case of multiplicity 2 ): $z=1$ and the roots of the high-degree polynomial,

$$
\begin{aligned}
s(t)= & 28025208984375+121442572265625 t+242885144531250 t^{2}+\left(290747126953125-51018336 G_{3}\right) t^{3} \\
& +\left(225342158203125-170061120 G_{3}\right) t^{4}+\left(113305394531250-160613280 G_{3}\right) t^{5} \\
& +\left(34211742187500+30792960 G_{3}\right) t^{6}+\left(4456582031250+113724000 G_{3}\right) t^{7} \\
& +\left(-433318359375+26827200 G_{3}\right) t^{8}+\left(-131466796875-24559200 G_{3}\right) t^{9} \\
& +\left(37300781250-9072000 G_{3}\right) t^{10}+\left(14291015625+1728000 G_{3}\right) t^{11}+\left(1263671875+768000 G_{3}\right) t^{12},
\end{aligned}
$$

that will be denoted by $s_{i}\left(G_{3}\right), i=1, \ldots, 12$.

Nevertheless, the complexity of the operator can be lower depending on the value of the parameter, as we can see in the following result, where we analyze when the number of strange fixed points decrease.

Theorem 6 The number of strange fixed points of operator $O_{q}\left(z, G_{3}\right)$ is thirteen (including $z=1$, for $G_{3} \neq-495868$ ), excluding the following cases:

(i) If $G_{3}=0$, the number of strange fixed points is nine, $z=1$ and the roots of $177147+531441 t+708588 t^{2}+512487 t^{3}+$ $187758 t^{4}+12663 t^{5}-10476 t^{6}-447 t^{7}+647 t^{8}$, that are complex.

(ii) For $G_{3}=\frac{1765625}{324}$, there are eleven strange fixed points, $z=1, z=-\frac{9}{5}$ (double) and the eight complex roots of polynomial $13286025+41039055 t+56529576 t^{2}+41862825 t^{3}+14979330 t^{4}+172665 t^{5}-1252800 t^{6}-33825 t^{7}+75325 t^{8}$. 
(iii) For $G_{3}=-\frac{41281250}{9}$, the strange fixed points are $z=1, z=-\frac{3}{5}$ (double), $z=\frac{3}{25}(3 \pm 8 \sqrt{6})$ and the (complex) roots of the polynomial $4428675+3838185 t+11626092 t^{2}+32057775 t^{3}+15658110 t^{4}-12474945 t^{5}-7325100 t^{6}+1911225 t^{7}+$ $1081775 t^{8}$.

As we will see in the following, not only the number but also the stability of the fixed points depend on the parameter of the family. The expression of the differential operator, necessary for analyzing the stability of the fixed points and for obtaining the critical points, can be easily obtained from the fixed point iteration function, $O_{q}\left(z, G_{3}\right)$, so it will be obviated.

On the other hand, let us remark that 0 is a superattractive fixed point and, as in case $m=2$, the stability of the other fixed points change depending on the values of the parameter $G_{3}$. In the following results we establish the stability of $z=\infty$ and the strange fixed point $z=1$, whose proofs are similar to that of Theorem 4 , and they are omitted.

Theorem 7 The stability of the fixed point $z=\infty$ is defined as follows:

(i) If $\left|-623046875-384000 G_{3}\right|<\frac{17578125}{2}$, then $z=\infty$ is attracting and superattracting for $G_{3}=-\frac{4984375}{3072}$.

(ii) For $\left|-623046875-384000 G_{3}\right|=\frac{17578125}{2}, z=\infty$ is a parabolic point.

(iii) If $\left|-623046875-384000 G_{3}\right|>\frac{17578125}{2}$, then $z=\infty$ is repulsive.

Theorem 8 The character of the strange fixed point $z=1$ is as follows (except for $G_{3}=-495868$, as in this case $z=1$ is not a fixed point):

(i) If $\left|7308593750+14739 G_{3}\right|>72 \cdot 10^{9}$, then $z=1$ is an attractor (it cannot be a superattractor).

(ii) When $\left|7308593750+14739 G_{3}\right|=72 \cdot 10^{9}, z=1$ is a parabolic point.

(iii) If $\left|7308593750+14739 G_{3}\right|<72 \cdot 10^{9}$, then $z=1$ is repulsive.

Let us remark that the area of the complex plane where $z=1$ is repulsive is even bigger than in case $m=2$ (see Figure 6). This makes the family even more stable, as the study of the stability of the rest of strange fixed points establishes.

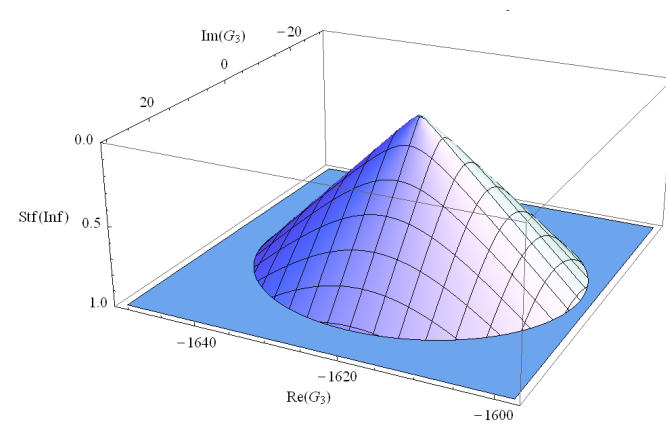

(a) $\left|\operatorname{Inf} f_{q}^{\prime}\left(0, G_{3}\right)\right|$

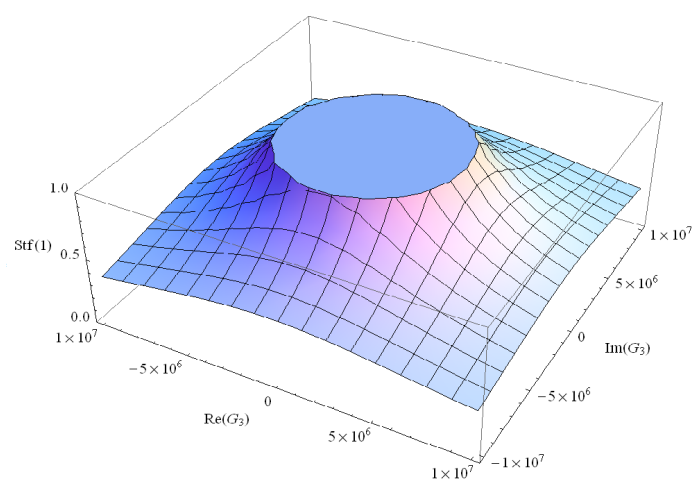

(b) $\left|O_{q}^{\prime}\left(1, G_{3}\right)\right|$

Fig. 6: Stability function of the fixed points $z=\infty$ and $z=1$, for $m=3$

Regarding the rest of strange fixed points, as it is not possible to get an analytical expression for them, we state both numerical and graphically, some general aspects on their stability: $s_{i}\left(G_{3}\right), i=1,2,3$ are repulsive for all values of $G_{3}$; the case of $s_{4}\left(G_{3}\right)$ is different, as it is superattracting for $G_{3}=-3014.73 \pm 1507.35 i$, and attracting in small cardioid around these values. Regarding $s_{5}\left(G_{3}\right)$ and $s_{6}\left(G_{3}\right)$, they are found also to be repulsive for any value of the parameter, meanwhile $s_{7}\left(G_{3}\right)$ is attracting in both cardioid around $G_{3}=-3012.96 \pm 1504.96 i$, being superattracting at these values. Again $s_{8}\left(G_{3}\right), s_{9}\left(G_{3}\right)$ and $s_{11}\left(G_{3}\right)$ are repulsive, whilst $s_{10}\left(G_{3}\right)$ is attracting inside a big area of the complex plane $\left[1 \cdot 10^{6}, 4.5 \cdot 10^{6}\right] \times\left[-2 \cdot 10^{6}, 2 \cdot 10^{6}\right]$, and superattracting at two points: $G_{3}=2.63009 \cdot 10^{6} \pm 370441 i$. Finally, $s_{12}\left(G_{3}\right)$ is superattracting if $G_{3}=-3052.6 \pm 1544.22 i$, being attracting in close complex values. Summarizing, there are no real values of $G_{3}$ where any of these strange fixed points is attracting or superattracting and their stability regions are very far from the origin.

This global behavior of the strange fixed points can be observed at Figure 7a, where the stability regions of $z=1$ and $z=\infty$ are also included. It is clear that the area around the origin is completely stable for the methods (as far as it concerns to fixed points of the rational operator), as the strange fixed points are repulsive. The rest of dangerous behavior is, moreover, very far from the standard values of a parameter in real applications. A final study, by using dynamical planes, is needed in order to see if the complex area surrounding the origin involves attracting periodic orbits for $m=3$, as it happened in the case of double roots. In order to understand more deeply the behavior of the elements of the family applied on polynomials 


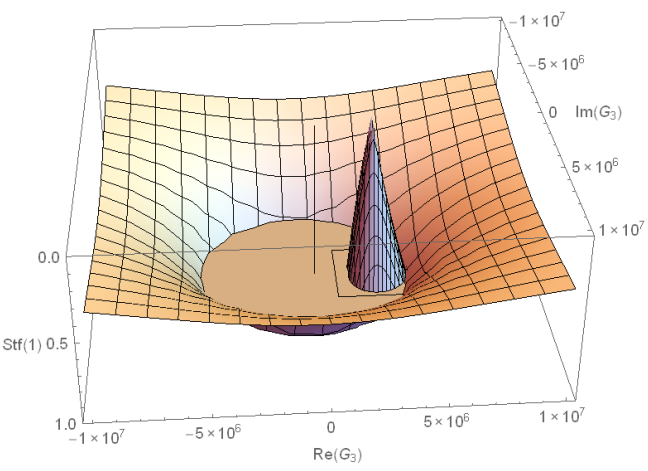

(a) $\left|O_{q}^{\prime}\left(s_{i}\left(G_{3}\right), G_{3}\right)\right|, i=2,3, \ldots, 12$

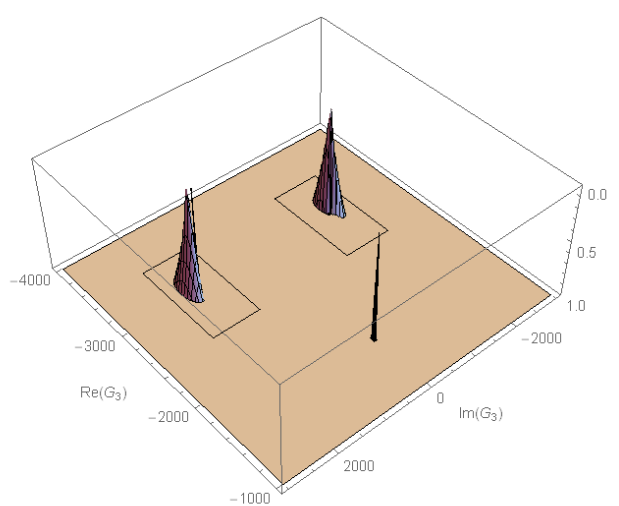

(b) A detail

Fig. 7: Stability functions of the strange fixed points $s_{i}\left(G_{3}\right), i=2,3, \ldots, 12$ for $m=3$

with cubic multiplicity, we analyze the number of critical points of the associated operator, as a lower number decreases the number of attracting areas different from the roots. In the following result these items are discussed, from the analysis of the equation $O_{q}^{\prime}\left(z, G_{3}\right)=0$.

Theorem 9 The free critical points of rational function $O_{q}\left(z, G_{3}\right)$ are $z=-m=-3$ (which is pre-image of $z=1$ ) and the roots $C_{i}\left(G_{3}\right), i=5,6, \ldots, 18$, of a 11th-polynomial, except in the following cases:

(i) If $G_{3}=0$, the only free critical points are $z=-3$ and the roots of $-231822-388071 t-126198 t^{2}+168885 t^{3}+$ $110142 t^{4}-3885 t^{5}-8346 t^{6}+319 t^{7}$.

(ii) For $G_{3}=-103515625 / 864 \approx 119810$, there are eleven free critical points, $z=-3$ and the roots of $57572775+$ $581868846 t+944584983 t^{2}+55611036 t^{3}-725941116 t^{4}-283149756 t^{5}+154120320 t^{6}+73364940 t^{7}-14681451 t^{8}-$ $6637338 t^{9}+209129 t^{10}$

(iii) If $G_{3} \approx-3036.44$ or $G_{3}=-118489$, there are eleven different free critical points, including $z=-3$, as $C_{2}\left(G_{3}\right)=$ $C_{3}\left(G_{3}\right)$.

(iv) For $G_{3}=-\frac{7308593750}{14739} \approx-495868$ there are ten free critical points, including $z=-3$.

(vi) If $G_{3} \approx-3341.1$ or $G_{3} \approx 3609.71$, the number of free critical points is eleven, one of them double.

These values of $G_{3}$ are candidates to stable values, as the number of possible basins of attraction decreases with the number of free critical points. So, the lower is this amount, lower is the probability to find stable behavior different from those of zero and infinity.

\subsubsection{Dynamical planes}

In this section, some of the values of $G_{3}$ that have appeared along this analysis are used to plot the respective dynamical planes and observe the performance of the method. In the studied cases, convergence to the multiple root $z=0$ or to both $z=0$ and $z=\infty$ are observed, depending on the value of the parameter. For the first case, see Figures $8 \mathrm{a}$ to $8 \mathrm{~d}$ where no attracting periodic orbits appears although the values of $G_{3}$ used are near the origin; regarding the former case, a dynamical plane for a superattracting $z=\infty$ is shown at Figure 8e, with a detail at Figure 8f.

There have been also appeared some values of $G_{3}$ corresponding to unstable behavior, under different circumstances: when we have analyzed the stability region of attracting strange fixed points (Figures $9 \mathrm{a}$ and $9 \mathrm{~b}$ for $z=1$ ), pairs of simultaneous attracting fixed points $\left(z=1\right.$ has bifurcated into $z_{1}=0.9859+0.3938 i$ and $z_{2}=0.9859-0.3938 i$ appearing in Figure $9 \mathrm{c}$ ), or $G_{3}$ being superattracting in Figure 9d; but also some of them correspond to attracting periodic orbits, as $\{0.8844 \pm 0.3098 i\}$ that can be seen at Figures 9e, or the case of $G_{3}=-3260-1500 i$, that has a black region as the basin of attraction of a high-period orbit (see Figure 9f).

\section{Numerical experiments}

In this section, we will check the efficiency and convergence behavior of our proposed schemes:

In this regards, we consider a total number of four test problems: first one is the trajectory of an electron in the air gap problem, second one is from the linear algebra; third and fourth are the concrete variety of standard test functions; fifth one is Van der Waals equation of state; sixth one is population growth problem, which are mentioned in the examples (1)- (6). 


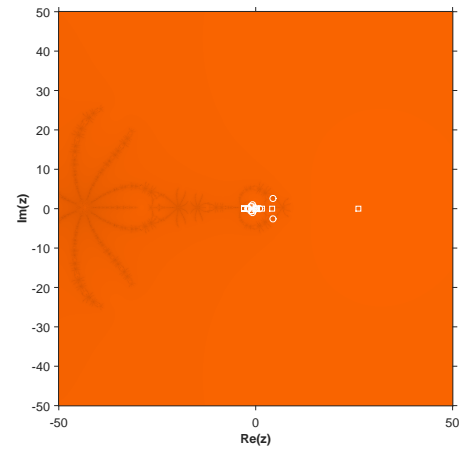

(a) $G_{3}=0$, a detail

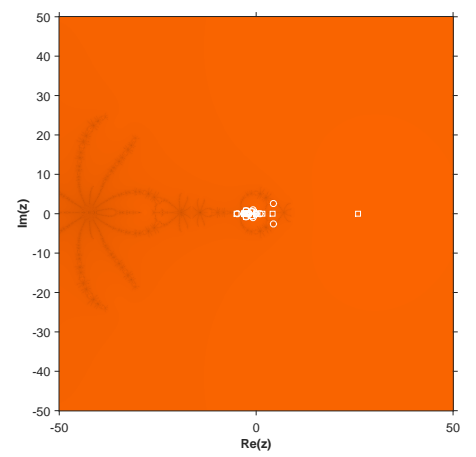

(d) $G_{3}=-50$

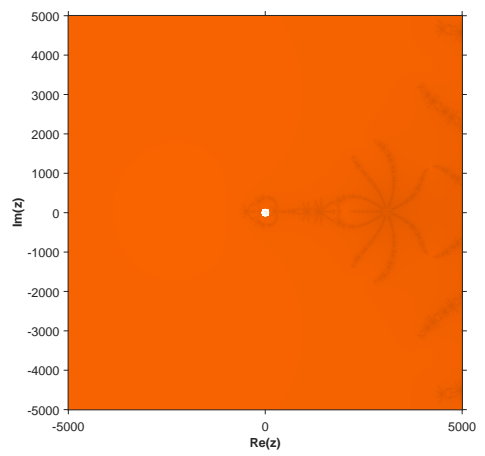

(b) $G_{3}=0$

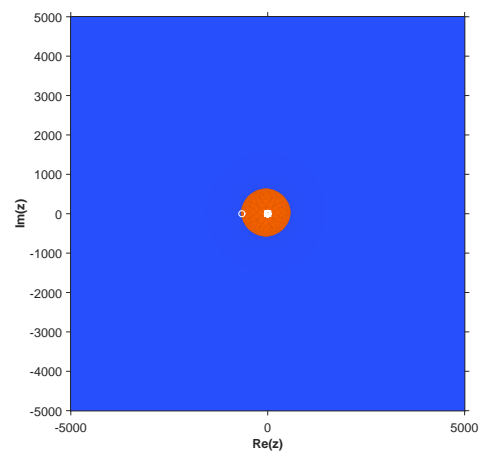

(e) $G_{3}=-\frac{4984375}{3072}$

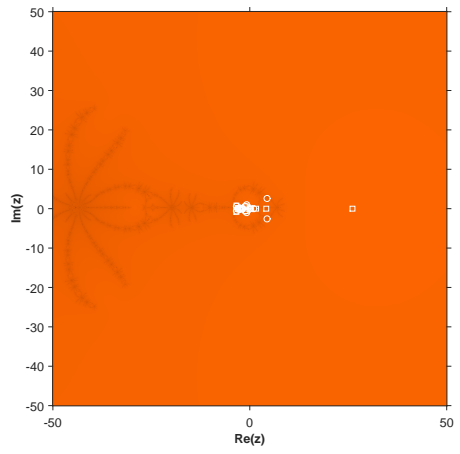

(c) $G_{3}=10$

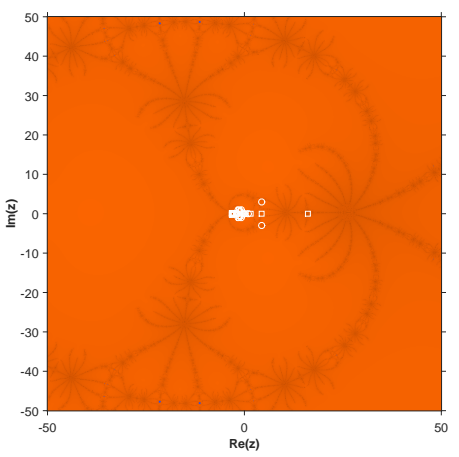

(f) $G_{3}=-\frac{4984375}{3072}$, a detail

Fig. 8: Dynamical planes with stable behavior for $m=3$

\begin{tabular}{|c|l|c|}
\hline Cases & Parameter & Method denoted by \\
\hline Case-1 & $\left.G_{3}=-\frac{m^{5}}{32 p^{3}}\left(m^{4}+2 m^{3}+2 m^{2}-2 m+12\right)\right)$ & $O M 1$ \\
Case-1 & $G_{3}=0$ & $O M 2$ \\
Case-2 & - & $O M 3$ \\
Case-3 & $a_{2}=\frac{p^{2}}{192} m(m+2)^{4}\left(m^{4}+4 m^{3}+6 m^{2}+2 m+8\right)$ & $O M 4$ \\
Case-4 & $a_{2}=\frac{p^{2}}{192} m(m+2)^{4}\left(m^{4}+4 m^{3}+6 m^{2}+2 m+8\right)$ & $O M 5$ \\
\hline
\end{tabular}

Now, we will compare our proposed methods with optimal families of fourth-order methods which were given by Hueso et al. in [6] and $\mathrm{Li}$ et al. [8], out of these families we shall choose expression (1) and expression (69), respectively called by $H M T$ and $L C N$. In addition, we compare them with fourth-order optimal schemes presented by Sharifi et al. [13] and Soleymani and Babajee [14], out of them we consider expression (35) and expression (27), respectively denoted by $S B S$ and $S B$. Moreover, we also compare them with robust and optimal schemes of the same order given by Soleymani et al. [15] and Zhou et al. [17], out of them we pick expression (18) and expression (27), respectively called by $S B L$ and $Z C S$. Further, we compare them with the optimal fourth-order family based on weight function approach presented by Zhou et al. [18], out of them we choose expression (11), denoted by $Z M$. Finally, we also compare them with the third-order scheme given by Sbibih et al. [11], out of them we choose expression (4.10) (for $\left.\mu=\frac{1}{3}\right)$ and (4.10) (for $\mu=\sqrt{2}$ ), called by $S A T Z 1$ and $S A T Z 2$, respectively.

For better comparison, we have compared our methods with them on the basis of approximated zeros, residual error of the involved functions, difference between the two consecutive iterations, asymptotic error constants. In Tables $2-7$, we displayed the number of iteration indexes $(n)$, approximated zeros $\left(x_{n}\right)$, absolute residual error of the corresponding function $\left(\left|f\left(x_{n}\right)\right|\right)$, error in the consecutive iterations $\left|x_{n+1}-x_{n}\right|,\left|\frac{x_{n+1}-x_{n}}{\left(x_{n}-x_{n-1}\right)^{4}}\right|$, the estimation of asymptotic error constant $\eta \approx \lim _{n \rightarrow \infty}\left|\frac{x_{n+1}-x_{n}}{\left(x_{n}-x_{n-1}\right)^{4}}\right|$ at the last iteration. We make our calculations with several number of significant digits (minimum 1000 significant digits) to minimize the round off error. 


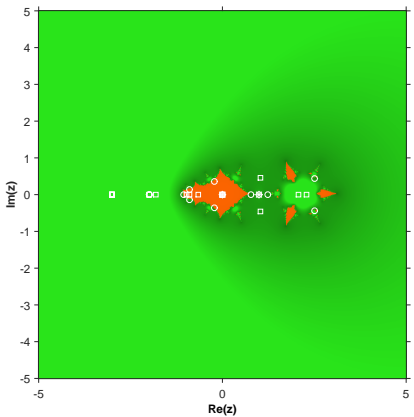

(a) $G_{3}=5 \cdot 10^{6}, z=1$ attracting

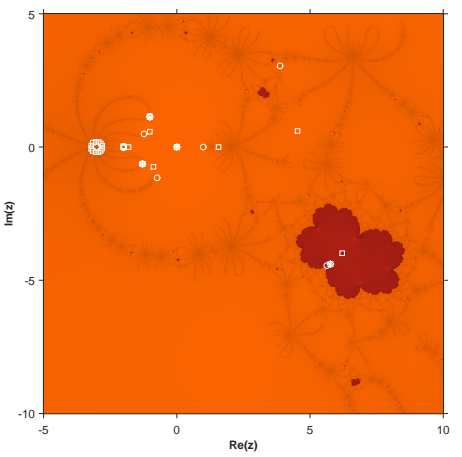

(d) $G_{3}=-3014.73-1507.35 i$

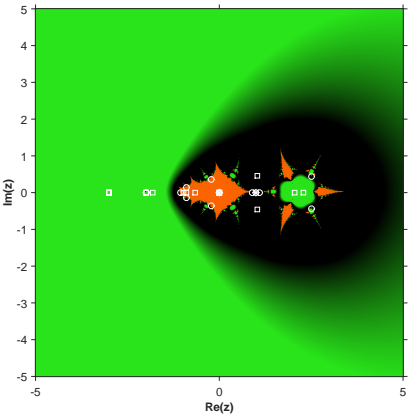

(b) $G_{3}=4.5 \cdot 10^{6}$

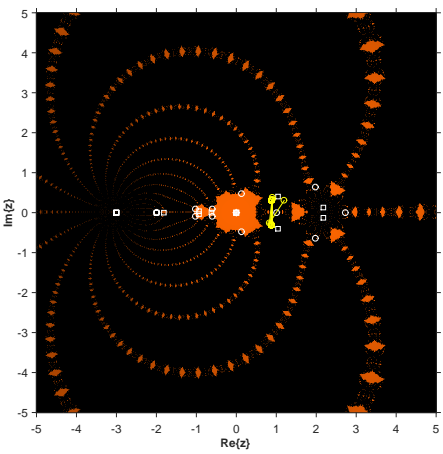

(e) $G_{3}=-4 \cdot 10^{6}$

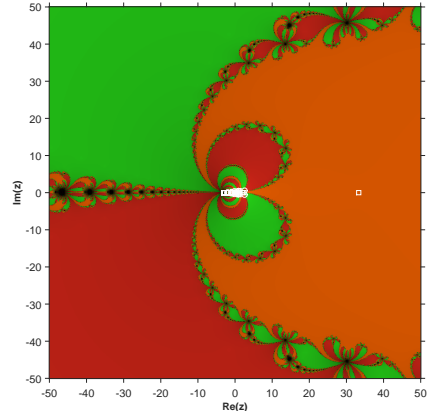

(c) $G_{3}=3 \cdot 10^{6}$

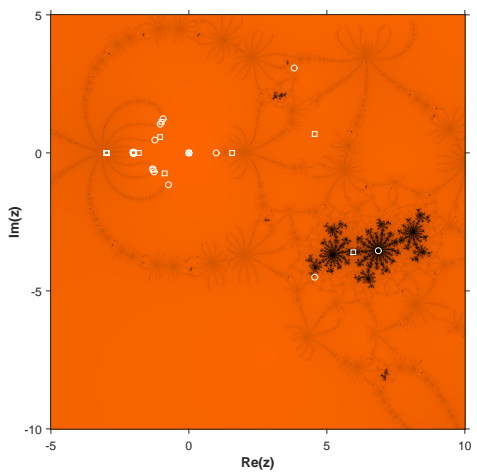

(f) $G_{3}=-3260-1500 i$

Fig. 9: Dynamical planes with unstable behavior for $m=3$

As we mentioned in the above paragraph that we calculate the values of all the constants and functional residuals up to several number of significant digits but due to the limited paper space, we display the value of $x_{n}$ up to 25 significant digits. In addition, we also display $\left|\frac{x_{n+1}-x_{n}}{\left(x_{n}-x_{n-1}\right)^{4}}\right|$ and $\eta$ up to 10 significant digits. Moreover, absolute residual error in the function $\left|f\left(x_{n}\right)\right|$ and error in the consecutive iterations $\left|x_{n+1}-x_{n}\right|$ are displayed up to 2 significant digits with exponent power which are mentioned in Tables $2-7$. Furthermore, the approximated zeros up to 25 significant digits are also displayed in the examples (1)- (6) although minimum 1000 significant digits are available with us.

For the computer programming, all computations have been performed using the programming package Mathematica 11 with multiple precision arithmetic. Further, the meaning of $a( \pm b)$ is $a \times 10^{( \pm b)}$ in the following Tables 2-7. Moreover, we also mentioned the CPU time in Table 8 which are calculated on the HP-Laptop, Windows 7 ultimate, RAM 4.00GB with 64-bit operating system.

Example 1 In the study of the multi-factor effect, the trajectory of an electron in the air gap between two parallel plates is given by

$$
x(t)=x_{0}+\left(v_{0}+e \frac{E_{0}}{m \omega} \sin \left(\omega t_{0}+\alpha\right)\right)\left(t-t_{0}\right)+e \frac{E_{0}}{m \omega^{2}}(\cos (\omega t+\alpha)+\sin (\omega+\alpha)),
$$

where $e$ and $m$ are the charge and the mass of the electron at rest, $x_{0}$ and $v_{0}$ are the position and velocity of the electron at time $t_{0}$ and $E_{0} \sin (\omega t+\alpha)$ is the RF electric field between the plates [6]. We choose the particular parameters in the expression (28) in order to deal with a simpler expression, which is defined as follows:

$$
f_{1}(x)=x+\cos (x)-\frac{\pi}{2}
$$

The above function has one multiple zero at $x=\frac{\pi}{2}$ of multiplicity three. 
Example 2 Consider the following $5 \times 5$ matrix

$$
B=\left[\begin{array}{ccccc}
29 & 14 & 2 & 6 & -9 \\
-47 & -22 & -1 & -11 & 13 \\
19 & 10 & 5 & 4 & -8 \\
-19 & -10 & -3 & -2 & 8 \\
7 & 4 & 3 & 1 & -3
\end{array}\right]
$$

The corresponding characteristic polynomial of this matrix is as follows:

$$
f_{2}(x)=(x-2)^{4}(x+1) \text {. }
$$

It's characteristic equation has one multiple root at $x=2$ of multiplicity four.

Example 3 Let us consider the following nonlinear equation chosen from [1]

$$
f_{3}(x)=\left(\cos \left(\frac{\pi x}{2}\right)-\sqrt{1-x^{2}}+x+1\right)^{3}
$$

The above function has one multiple zero at $x=-0.7285840464448267167123331$ of multiplicity three.

Example 4 Let us pick a standard nonlinear test function from [6], which is given as follows:

$$
f_{4}(x)=\exp (x)-\sum_{k=0}^{l-1} \frac{x^{k}}{k !},
$$

where $l=20$. The above function has a multiple root at $x=0$ of multiplicity $l$.

\section{Example 5 Van der Waals equation of state:}

$$
\left(P+\frac{a_{1} n^{2}}{V^{2}}\right)\left(V-n a_{2}\right)=n R T,
$$

explains the behavior of a real gas by introducing in the ideal gas equations two parameters, $a_{1}$ and $a_{2}$, specific for each gas. The determination of the volume $V$ of the gas in terms of the remaining parameters requires the solution of a nonlinear equation in $\mathrm{V}$,

$$
P V^{3}-\left(n a_{2} P+n R T\right) V^{2}+a_{1} n^{2} V-a_{1} a_{2} n^{2}=0 .
$$

Given the constants $a_{1}$ and $a_{2}$ of a particular gas, one can find values for $n, P$ and $T$, such that this equation has three simple roots. By using the particular values, we obtain the following nonlinear function

$$
f_{5}(x)=x^{3}-5.22 x^{2}+9.0825 x-5.2675 .
$$

which has three zeros and out of them one is the multiple zero $x=1.75$ of multiplicity 2 , and the other is the simple zero $x=1.72$. However, our desired zero is $x=1.75$.

\section{Example 6 Population growth problem:}

Law of population growth is defined as follows:

$$
\frac{d N(t)}{d t}=\gamma N(t)+\eta
$$

where $\mathrm{N}(\mathrm{t})=$ population at time $t, \eta=$ fixed/constant immigration rate and $\gamma=$ fixed/constant birth rate of population. We can easily obtain the following solution of the above differential equation (36)

$$
N(t)=N_{0} e^{\gamma t}+\frac{\eta}{\gamma}\left(e^{\gamma t-1}\right)
$$

where $N_{0}$ is initial population.

For a particular case study, the problem is given as: Suppose a certain population contains 1000000 individuals initially, that 300000 individuals immigrate into the community in the first year and that 1365000 individuals are present at the end of one year. Find birth rate $(\gamma)$ of this population.

To determine the birth rate, we must solve the equation

$$
f_{6}(x)=1365-1000 e^{x}-\frac{300}{x}\left(e^{x}-1\right) .
$$

wherein $x=\gamma$ and our desired zero of the above function $f_{6}$ is 0.05504622451335177827483421 . 
Remark 1 It is worthy to note from the Tables 2-7 that our proposed methods are efficient for determining multiple roots of nonlinear equations and are competitive with other well recognized efficient and optimal fourth-order iterative methods, being $O M 1$ and $O M 4$ those obtaining better results in the most of problems analyzed. In addition, our methods have not only minimum residual error corresponding to considered test function $f$ but also have small asymptotic error constant in most of the considered test functions. Moreover, minimum error between the consecutive iterations corresponding to the considered functions belongs to our proposed iterative methods. Hence, we confirm that our methods converge faster towards required zero of the corresponding function as compared to other existing methods.

The comparison among different schemes can be made numerically, by using certain initial estimations as we have made in Tables 2-5, or graphically by means of their basins of attraction. In them, we are going to obtain the set of converging initial points for all the optimal methods considered and functions $f_{1}(x)$ and $f_{2}(x)$. As this suppose 24 images, we consider it is enough with this two functions. As in Section 3, these dynamical planes have been obtained by using a mesh of $800 \times 800$ points, a tolerance of $10^{-3}$ and 200 as maximum number of iterations. In them, we paint in blue color the points whose orbits diverge, in orange, green,... the convergent points to the roots and in black those initial guesses that do not converge with 200 iterations.

From the basins of attraction presented in Figure 10, it can be observed that LCN, SBL and OM3 methods do not present divergent regions, that appears in the corresponding dynamical planes of HMT, ZCS, ZM, SB and OM2 with wide basins of attraction of the multiple root. However, methods SBS and OM5 show smaller areas of convergence to the multiple root.

In Figure 11 the dynamical behavior of the compared methods on $f_{2}(x)$ appear. Many of them (HMT, SB, ZCS, ZM, OM1, OM2, OM4) show global convergence (in the analyzed area of the complex plane), with slightly dark areas of slower convergence, and again SBS and OM5 present worse results, with areas of divergence and small basins of attraction to the multiple root.

\section{Concluding remarks}

In the earlier studies, several scholars proposed higher-order optimal fourth-order iterative methods for multiple roots when the multiplicity $m$ is known in advance. But most of these schemes have the flexibility only at the second step due to the involvement of weight function/s or free parameter/s in order to explore other new schemes. Our mean to say that if a researcher want to explore some more methods from the same scheme then he/she can make changes only at second step not at the first step for example please see all the mentioned methods (whose first step is fixed) in the introduction section. Therefore, we contribute further to the development of the theory of iteration processes and propose a new optimal fourth-order scheme in a simple way based on mid-point and weight function approach. The main beauty of our proposed scheme is that they have not only given the flexibility to the researchers at both steps in order to construct a new optimal fourth-order methods but also give the faster convergence, small residual error corresponding to the involved function and asymptotic error constants.

The proposed scheme is optimal in the sense of classical Kung-Traub conjecture. We also compare our methods with the existing robust methods of same order on a series of numerical examples. The results in Table 2 and 7 overwhelmingly support that the minimum residual errors, minimum error in the consecutive iterations and small asymptotic error constants belongs to our proposed methods. In addition, the study of dynamics of our methods also reflects that there are wide areas of stability corresponding to values of $G_{3}$ greater than two hundred units in absolute value, but it is also stable in other many cases. The superiority of our methods over the existing robust methods may be due to the inherent structure of our methods with mid point and weight function approach. The future work based on the weight function approach shall be devoted to the construction of a new optimal higher-order methods.

\section{Acknowledgments}

The authors would like to thank the anonymous reviewers for their suggestions that have improved the final version of this manuscript.

\section{References}

1. R. Behl, A. Cordero, S.S. Motsa, J.R. Torregrosa, V. Kanwar, An optimal fourth-order family of methods for multiple roots and its dynamics. Numer. Algor. 71 (4) (2016) 775-796.

2. P. Blanchard, Complex analytic dynamics on the Riemann sphere, Bull. Amer. Math. Soc. 11(1) (1984) 85-141.

3. F. Chicharro, A. Cordero, J.R. Torregrosa, Drawing dynamical and parameter planes of iterative families and methods, Sci. World J. 2013 (2013) Article ID 78015311 pages.

4. R.L. Devaney, The Mandelbrot Set, the Farey Tree and the Fibonacci sequence, American Mathematical Monthly 106(4) (1999) $289-302$. 


\begin{tabular}{|c|c|c|c|c|c|c|}
\hline Cases & $n$ & $x_{n}$ & $\left|f\left(x_{n}\right)\right|$ & $\left|x_{n+1}-x_{n}\right|$ & \begin{tabular}{|l|}
$x_{n+1}-x_{n}$ \\
$\left(x_{n}-x_{n-1}\right)^{4}$
\end{tabular} & $\eta$ \\
\hline \multirow{4}{*}{$H M T$} & 0 & 1 & $3.0(-2)$ & $5.7(-1)$ & & \\
\hline & 1 & 1.570873296646599848130845 & $7.6(-14)$ & $7.7(-5)$ & $7.247057960(-4)$ & \\
\hline & 2 & 1.570796326794896619231318 & $5.8(-72)$ & $3.3(-24)$ & $9.309686833(-8)$ & \\
\hline & 3 & 1.570796326794896619231322 & $1.5(-362)$ & $4.5(-121)$ & $3.952137931(-27)$ & $3.952137931(-27)$ \\
\hline \multirow{4}{*}{$L C N$} & 0 & 1 & $3.0(-2)$ & $5.7(-1)$ & & \\
\hline & 1 & 1.570873174238979227550361 & $7.6(-14)$ & $7.7(-5)$ & $7.235538937(-4)$ & \\
\hline & 2 & 1.570796326794896619231318 & $5.7(-72)$ & $3.2(-24)$ & $9.294881340(-8)$ & \\
\hline & 3 & 1.570796326794896619231322 & $1.4(-362)$ & $1.3(-121)$ & $3.920811670(-27)$ & $3.920811670(-27)$ \\
\hline \multirow{4}{*}{$S B S$} & 0 & 1 & $3.0(-2)$ & $6.2(-1)$ & & \\
\hline & 1 & 1.616870294223802341069037 & $1.6(-5)$ & $4.6(-2)$ & $3.181864081(-1)$ & \\
\hline & 2 & 1.570796173901997451491397 & $6.0(-22)$ & $1.5(-7)$ & $3.392806624(-2)$ & \\
\hline & 3 & 1.570796326794896619231322 & $3.9(-104)$ & $6.2(-35)$ & $1.125657728(-8)$ & $1.125657728(-8)$ \\
\hline \multirow{4}{*}{$S B$} & 0 & 1 & $3.0(-2)$ & $5.7(-1)$ & & \\
\hline & 1 & 1.570872980187187161201669 & $7.5(-14)$ & $7.7(-5)$ & $7.217277885(-4)$ & \\
\hline & 2 & 1.570796326794896619231318 & $5.5(-72)$ & $3.2(-24)$ & $9.271410313(-8)$ & \\
\hline & 3 & 1.570796326794896619231322 & $1.1(-362)$ & $4.1(-121)$ & $3.871557739(-27)$ & $3.871557739(-27)$ \\
\hline \multirow{4}{*}{$S B L$} & 0 & 1 & $3.0(-2)$ & $5.7(-1)$ & & \\
\hline & 1 & 1.570873282324298463651858 & $7.6(-14)$ & $7.7(-5)$ & $7.245710178(-4)$ & \\
\hline & 2 & 1.570796326794896619231318 & $5.8(-72)$ & $3.3(-24)$ & $9.307954517(-8)$ & \\
\hline & 3 & 1.570796326794896619231322 & $1.5(-362)$ & $4.5(-121)$ & $3.948462294(-27)$ & $3.948462294(-27)$ \\
\hline \multirow{4}{*}{$Z C S$} & 0 & 1 & $3.0(-2)$ & $5.8(-1)$ & & \\
\hline & 1 & $1.580185516+5.643239597(-3) i$ & $2.2(-7)$ & $1.1(-2)$ & $9.666047943(-2)$ & \\
\hline & 2 & $1.570796292-6.763923186(-8) i$ & $7.3(-23)$ & $7.6(-8)$ & 5.270320098 & \\
\hline & 3 & $1.570796327+9.874665011(-40) i$ & $4.6(-118)$ & $1.4(-39)$ & $4.216567639(-11)$ & $4.216567639(-11)$ \\
\hline \multirow{4}{*}{$Z M$} & 0 & 1 & $3.0(-2)$ & $5.7(-1)$ & & \\
\hline & 1 & 1.570873332024493686354515 & $7.6(-14)$ & $7.7(-5)$ & $7.250387150(-4)$ & \\
\hline & 2 & 1.570796326794896619231318 & $5.9(-72)$ & $3.3(-24)$ & $9.313965874(-8)$ & \\
\hline & 3 & 1.570796326794896619231322 & $1.6(-362)$ & $4.6(-121)$ & $3.961228952(-27)$ & $3.961228952(-27)$ \\
\hline \multirow{4}{*}{$S A T Z 1$} & 0 & 1 & $3.0(-2)$ & $5.7(-1)$ & & \\
\hline & 1 & 1.567866295736378901239945 & $4.2(-9)$ & $2.9(-3)$ & $2.817654874(-2)$ & \\
\hline & 2 & 1.570796326422235906422354 & $8.6(-30)$ & $3.7(-10)$ & 5.056208048 & \\
\hline & 3 & 1.570796326794896619231322 & $7.5(-92)$ & $7.7(-31)$ & $3.975416325(+7)$ & $3.975416325(+7)$ \\
\hline \multirow{4}{*}{$S A T Z 2$} & 0 & 1 & $3.0(-2)$ & $5.7(-1)$ & & \\
\hline & 1 & 1.569081716959350346276960 & $8.4(-10)$ & $1.7(-3)$ & $1.634808047(-2)$ & \\
\hline & 2 & 1.570796326750487893968856 & $1.5(-32)$ & $4.4(-11)$ & 5.138153914 & \\
\hline & 3 & 1.570796326794896619231322 & $7.7(-101)$ & $7.7(-34)$ & $1.983827413(+8)$ & $1.983827413(+8)$ \\
\hline \multirow{4}{*}{$O M 1$} & 0 & 1 & $3.0(-2)$ & $5.7(-1)$ & & \\
\hline & 1 & 1.570869165130719723566698 & $6.4(-14)$ & $7.3(-5)$ & $6.858255680(-4)$ & \\
\hline & 2 & 1.570796326794896619231319 & $2.5(-72)$ & $2.5(-24)$ & $8.809970141(-8)$ & \\
\hline & 3 & 1.570796326794896619231322 & $2.4(-364)$ & $1.1(-121)$ & $2.999362222(-27)$ & $2.999362222(-27)$ \\
\hline \multirow{4}{*}{$O M 2$} & 0 & 1 & $3.0(-2)$ & $5.7(-1)$ & & \\
\hline & 1 & 1.570873461348743074737537 & $7.6(-14)$ & $7.7(-5)$ & $7.262557026(-4)$ & \\
\hline & 2 & 1.570796326794896619231318 & $6.0(-72)$ & $3.3(-24)$ & $9.329607950(-8)$ & \\
\hline & 3 & 1.570796326794896619231322 & $1.8(-362)$ & $1.4(-121)$ & $3.994603733(-27)$ & $3.994603733(-27)$ \\
\hline \multirow{4}{*}{$O M 3$} & 0 & 1 & $3.0(-2)$ & $5.7(-1)$ & & \\
\hline & 1 & 1.570873139426480793294248 & $7.6(-14)$ & $7.7(-5)$ & $7.232262945(-4)$ & \\
\hline & 2 & 1.570796326794896619231318 & $5.6(-72)$ & $3.2(-24)$ & $9.290670685(-8)$ & \\
\hline & 3 & 1.570796326794896619231322 & $1.3(-362)$ & $4.3(-121)$ & $3.911938919(-27)$ & $3.911938919(-27)$ \\
\hline \multirow{4}{*}{$O M 4$} & 0 & 1 & $3.0(-2)$ & $5.7(-1)$ & & \\
\hline & 1 & 1.570869189427903515905001 & $6.4(-14)$ & $7.3(-5)$ & $6.860542268(-4)$ & \\
\hline & 2 & 1.570796326794896619231319 & $2.6(-72)$ & $2.5(-24)$ & $8.812908944(-8)$ & \\
\hline & 3 & 1.570796326794896619231322 & $2.5(-364)$ & $1.8(-121)$ & $3.004368150(-27)$ & $3.004368150(-27)$ \\
\hline \multirow{4}{*}{$O M 5$} & 0 & 1 & $3.0(-2)$ & $5.7(-1)$ & & \\
\hline & 1 & 1.585352159610620619427337 & $9.7(-15)$ & $3.9(-5)$ & $3.654802206(-4)$ & \\
\hline & 2 & 1.570796326794896619231322 & $2.0(-76)$ & $2.5(-24)$ & $4.693762169(-8)$ & \\
\hline & 3 & 1.570796326794896619231322 & $7.5(-385)$ & $1.8(-128)$ & $1.287544167(-28)$ & $1.287544167(-28)$ \\
\hline
\end{tabular}

Table 2: Convergence behavior of different higher-order iterative methods on the test function $f_{1}(x)$

5. Dong, C., A Family of Multipoint Iterative Functions for Finding Multiple Roots of Equations, Inter. J. Computer Math. 21(1987) 363-367

6. Hueso, J.L., Martínez E., Teruel, C.: Determination of multiple roots of nonlinear equations and applications. J. Math. Chem. 53 (2015) 880-892. 


\begin{tabular}{|c|c|c|c|c|c|c|}
\hline Cases & $n$ & $x_{n}$ & $\left|f\left(x_{n}\right)\right|$ & $\left|x_{n+1}-x_{n}\right|$ & $\frac{x_{n+1}-x_{n}}{\left(x_{n}-x_{n-1}\right)^{4}}$ & $\eta$ \\
\hline \multirow{4}{*}{$H M T$} & 0 & 1.5 & $1.6(-1)$ & $5.0(-1)$ & & \\
\hline & 1 & 2.000605976427265857393486 & $4.0(-13)$ & $6.1(-4)$ & $9.648762353(-3)$ & \\
\hline & 2 & 2.000000000000000674773199 & $6.2(-61)$ & $6.7(-16)$ & $5.004202823(-3)$ & \\
\hline & 3 & 2.000000000000000000000000 & $3.5(-252)$ & $1.0(-63)$ & $5007802704(-3)$ & $5.007802704(-3)$ \\
\hline \multirow{4}{*}{$L C N$} & 0 & 1.5 & $1.6(-1)$ & $5.0(-1)$ & & \\
\hline & 1 & 2.000598258969828368317889 & $3.8(-13)$ & $6.0(-4)$ & $9.526467264(-3)$ & \\
\hline & 2 & 2.000000000000000632154766 & $4.8(-61)$ & $6.3(-16)$ & $4.934765741(-3)$ & \\
\hline & 3 & 2.000000000000000000000000 & $1.2(-252)$ & $7.9(-64)$ & $4.938271605(-3)$ & $4.938271605(-3)$ \\
\hline \multirow{4}{*}{$S B S$} & 0 & 1.5 & $1.6(-1)$ & $5.0(-1)$ & & \\
\hline & 1 & 2.019404110787400172282947 & $4.3(-7)$ & $1.9(-2)$ & $2.666074196(-1)$ & \\
\hline & 2 & 1.999999999285862929544783 & $7.8(-37)$ & $7.1(-10)$ & $5.037401086(-3)$ & \\
\hline & 3 & 2.000000000000000000000000 & $7.6(-156)$ & $1.3(-39)$ & $4.854606300(-3)$ & $4.854606300(-3)$ \\
\hline \multirow{4}{*}{$S B$} & 0 & 1.5 & $1.6(-1)$ & $5.0(-1)$ & & \\
\hline & 1 & 2.000580803698747068224322 & $3.4(-13)$ & $5.8(-4)$ & $9.249805665(-3)$ & \\
\hline & 2 & 2.000000000000000542909459 & $2.6(-61)$ & $5.4(-16)$ & $4.771005473(-3)$ & \\
\hline & 3 & 2.000000000000000000000000 & $8.9(-254)$ & $4.1(-64)$ & $4.774305556(-3)$ & $4.774305556(-3)$ \\
\hline \multirow{4}{*}{$S B L$} & 0 & 1.5 & $1.6(-1)$ & $5.0(-1)$ & & \\
\hline & 1 & 2.000616459055436191733497 & $4.3(-13)$ & $6.2(-4)$ & $9.814851663(-3)$ & \\
\hline & 2 & 2.000000000000000737702294 & $8.9(-61)$ & $7.4(-16)$ & $5.108156426(-3)$ & \\
\hline & 3 & 2.000000000000000000000000 & $1.6(-251)$ & $1.5(-63)$ & $5.111882716(-3)$ & $5.111882716(-3)$ \\
\hline \multirow{4}{*}{$Z C S$} & 0 & 1.5 & $1.6(-1)$ & $5.6(-1)$ & & \\
\hline & 1 & 2.058579987081456363563905 & $3.6(-5)$ & $5.9(-2)$ & $6.017380565(-1)$ & \\
\hline & 2 & 2.000000041266657660868987 & $8.7(-30)$ & $4.1(-8)$ & $3.504317381(-3)$ & \\
\hline & 3 & 2.000000000000000000000000 & $4.2(-128)$ & $1.1(-32)$ & $3.761573884(-3)$ & $3.761573884(-3)$ \\
\hline \multirow{4}{*}{$Z M$} & 0 & 1.5 & $1.6(-1)$ & $5.0(-1)$ & & \\
\hline & 1 & 2.000606834142187230960775 & $4.1(-13)$ & $6.1(-4)$ & $9.662353244(-3)$ & \\
\hline & 2 & 2.000000000000000679634847 & $6.4(-61)$ & $6.8(-16)$ & $5.011821661(-3)$ & \\
\hline & 3 & 2.000000000000000000000000 & $3.9(-252)$ & $1.1(-63)$ & $5.015432099(-3)$ & $5.015432099(-3)$ \\
\hline \multirow{4}{*}{$S A T Z 1$} & 0 & 1.5 & $1.6(-1)$ & $5.0(-1)$ & & \\
\hline & 1 & 1.99546183624086726798384 & $1.3(-9)$ & $4.5(-3)$ & $7.530765614(-2)$ & \\
\hline & 2 & 1.999999997900536487365499 & $5.8(-35)$ & $2.1(-9)$ & 4.949809464 & \\
\hline & 3 & 2.000000000000000000000000 & $5.5(-111)$ & $2.1(-28)$ & $1.065757241(+7)$ & $1.065757241(+7)$ \\
\hline \multirow{4}{*}{$S A T Z 2$} & 0 & 1.5 & $1.6(-1)$ & $5.0(-1)$ & & \\
\hline & 1 & 1.996865833985800502425354 & $2.9(-10)$ & $3.1(-3)$ & $5.142394482(-2)$ & \\
\hline & 2 & 1.999999999491570721895109 & $2.0(-37)$ & $5.1(-10)$ & 5.269177705 & \\
\hline & 3 & 2.000000000000000000000000 & $6.6(-119)$ & $2.2(-30)$ & $3.240378726(+7)$ & $3.240378726(+7)$ \\
\hline \multirow{4}{*}{$O M 1$} & 0 & 1.5 & $1.6(-1)$ & $5.0(-1)$ & & \\
\hline & 1 & 2.000033487649437063732799 & $3.8(-18)$ & $3.3(-5)$ & $5.356588729(-4)$ & \\
\hline & 2 & 2.000000000000000000000000 & $1.3(-105)$ & $4.6(-27)$ & $3.634432827(-9)$ & \\
\hline & 3 & 2.000000000000000000000000 & $6.6(-543)$ & $2.2(-136)$ & $4.959442267(-31)$ & $4.959442267(-31)$ \\
\hline \multirow{4}{*}{$O M 2$} & 0 & 1.5 & $1.6(-1)$ & $5.0(-1)$ & & \\
\hline & 1 & 2.000612301010062421692316 & $4.2(-13)$ & $6.1(-4)$ & $9.748973916(-3)$ & \\
\hline & 2 & 2.000000000000000711227655 & $7.7(-61)$ & $7.1(-16)$ & $5.059979203(-3)$ & \\
\hline & 3 & 2.000000000000000000000000 & $8.5(-252)$ & $1.3(-61)$ & $5.063657407(-3)$ & $5.063657407(-3)$ \\
\hline \multirow{4}{*}{$O M 3$} & 0 & 1.5 & $1.6(-1)$ & $5.0(-1)$ & & \\
\hline & 1 & 2.000596167557409000156910 & $3.8(-13)$ & $6.0(-4)$ & $9.493322987(-3)$ & \\
\hline & 2 & 2.000596167557409000156910 & $4.5(-61)$ & $6.2(-16)$ & $4.915500737(-3)$ & \\
\hline & 3 & 2.000000000000000000000000 & $8.6(-253)$ & $7.3(-64)$ & $4.918981481(-3)$ & $4.918981481(-3)$ \\
\hline \multirow{4}{*}{$O M 4$} & 0 & 1.5 & $1.6(-1)$ & $5.0(-1)$ & & \\
\hline & 1 & 2.000042987177578592553261 & $1.0(-17)$ & $4.3(-5)$ & $6.875583612(-4)$ & \\
\hline & 2 & 2.000000000000000000000000 & $1.4(-105)$ & $4.6(-27)$ & $1.351547525(-9)$ & \\
\hline & 3 & 2.000000000000000000000000 & $5.7(-545)$ & $6.6(-137)$ & $1.452872236(-31)$ & $1.452872236(-31)$ \\
\hline \multirow{4}{*}{$O M 5$} & 0 & 1.5 & $1.6(-1)$ & $5.0(-1)$ & & \\
\hline & 1 & 2.001150840221343121297670 & $5.3(-12)$ & $1.2(-3)$ & $1.824488711(-2)$ & \\
\hline & 2 & 1.999999999999999999938591 & $4.3(-77)$ & $6.1(-20)$ & $3.500850225(-8)$ & \\
\hline & 3 & 2.000000000000000000000000 & $4.7(-402)$ & $2.7(-101)$ & $1.933187756(-28)$ & $1.933187756(-28)$ \\
\hline
\end{tabular}

Table 3: Convergence behavior of different higher-order iterative methods on the test function $f_{2}(x)$

7. Kung, H.T., Traub, J. F.: Optimal order of one-point and multipoint iteration. J. Assoc. Comput. Mach. 21 (1974) $643-651$.

8. Li, S.G., Cheng, L.Z., Neta, B.: Some fourth-order nonlinear solvers with closed formulae for multiple roots. Comput. Math. Appl. 59 (2010) 126-135. 


\begin{tabular}{|c|c|c|c|c|c|c|}
\hline Cases & $n$ & $x_{n}$ & $\left|f\left(x_{n}\right)\right|$ & $\left|x_{n+1}-x_{n}\right|$ & \begin{tabular}{|l|}
$x_{n+1}-x_{n}$ \\
$\left(x_{n}-x_{n-1}\right)^{4}$ \\
\end{tabular} & $\eta$ \\
\hline \multirow{4}{*}{$H M T$} & 0 & -0.3 & $2.6(-1)$ & $4.3(-1)$ & & \\
\hline & 1 & -0.7307541860524007798228250 & $2.6(-8)$ & $2.2(-3)$ & $6.303324668(-2)$ & \\
\hline & 2 & -0.7285840462944663849955303 & $8.7(-30)$ & $1.5(-10)$ & 6.779262665 & \\
\hline & 3 & -0.7285840464448267167123331 & $9.6(-116)$ & $3.4(-39)$ & 6.564620567 & 6.564620567 \\
\hline \multirow{4}{*}{$L C N$} & 0 & -0.3 & $2.6(-1)$ & $4.3(-1)$ & & \\
\hline & 1 & -0.7307410231568425088556431 & $2.5(-8)$ & $2.2(-3)$ & $6.265857938(-2)$ & \\
\hline & 2 & -0.7285840462998300981367198 & $7.8(-30)$ & $1.4(-10)$ & 6.698474873 & \\
\hline & 3 & -0.7285840464448267167123331 & $6.0(-116)$ & $2.9(-39)$ & 6.488701515 & 6.488701515 \\
\hline \multirow{4}{*}{$S B S$} & 0 & -0.3 & $2.6(-1)$ & $4.4(-1)$ & & \\
\hline & 1 & -0.7435562826197813060968027 & $8.3(-6)$ & $1.5(-2)$ & $3.868152117(-2)$ & \\
\hline & 2 & -0.7285836390969679989218396 & $1.7(-19)$ & $4.1(-7)$ & 8.105344879 & \\
\hline & 3 & -0.7285840464448267167123329 & $1.4(-74)$ & $1.8(-25)$ & 6.454556778 & 6.454556778 \\
\hline \multirow{4}{*}{$S B$} & 0 & -0.3 & $2.6(-1)$ & $4.3(-1)$ & & \\
\hline & 1 & -0.7307208210268841876594601 & $2.5(-8)$ & $2.1(-3)$ & $6.208336848(-2)$ & \\
\hline & 2 & -0.7285840463078707273473289 & $6.6(-30)$ & $1.4(-10)$ & 6.569708234 & \\
\hline & 3 & -0.7285840464448267167123331 & $2.9(-116)$ & $2.2(-39)$ & 6.367386517 & 6.367386517 \\
\hline \multirow{4}{*}{$S B L$} & 0 & -0.3 & $2.6(-1)$ & $4.3(-1)$ & & \\
\hline & 1 & -0.7307520405562654192284585 & $2.6(-8)$ & $2.2(-3)$ & $6.297218380(-2)$ & \\
\hline & 2 & -0.7285840462952534902103386 & $8.5(-30)$ & $1.5(-10)$ & 6.770509459 & \\
\hline & 3 & -0.7285840464448267167123331 & $9.0(-116)$ & $3.3(-39)$ & 6.556601701 & 6.556601701 \\
\hline \multirow{4}{*}{$Z C S$} & 0 & -0.3 & $2.6(-1)$ & $4.7(-1)$ & & \\
\hline & 1 & $-0.7668910299-2.477092481(-2) i$ & $2.2(-4)$ & $5.0(-2)$ & 1.036631767 & \\
\hline & 2 & $-0.7257871544+2.876282902(-3) i$ & $1.7(-7)$ & $4.0(-3)$ & 666.2561452 & \\
\hline & 3 & $-0.7285840474-5.368971121(-12) i$ & $2.3(-27)$ & $9.7(-10)$ & 3.733460864 & 3.733460864 \\
\hline \multirow{4}{*}{$Z M$} & 0 & -0.3 & $2.6(-1)$ & $4.3(-1)$ & & \\
\hline & 1 & -0.7307580515956084366130089 & $2.6(-8)$ & $2.2(-3)$ & $6.314325758(-2)$ & \\
\hline & 2 & -0.7285840462928719843464066 & $9.0(-30)$ & $1.5(-10)$ & 6.802551427 & \\
\hline & 3 & -0.7285840464448267167123331 & $1.1(-115)$ & $3.5(-39)$ & 6.586477782 & 6.586477782 \\
\hline \multirow{4}{*}{$S A T Z 1$} & 0 & -0.3 & $2.6(-1)$ & $4.3(-1)$ & & \\
\hline & 1 & -0.6840561491117837743108155 & $2.5(-4)$ & $4.4(-2)$ & 2.038634112 & \\
\hline & 2 & -0.7284086567042481427919098 & $1.4(-11)$ & $1.8(-4)$ & $4.532433387(+2)$ & \\
\hline & 3 & -0.7285840464286379883370366 & $1.1(-32)$ & $1.6(-11)$ & $1.710787836(+4)$ & $1.710787836(+4)$ \\
\hline \multirow{4}{*}{$S A T Z 2$} & 0 & -0.3 & $2.6(-1)$ & $4.0(-1)$ & & \\
\hline & 1 & -0.7025172838401661250476173 & $4.8(-5)$ & $2.6(-2)$ & $9.919969134(-1)$ & \\
\hline & 2 & -0.7285577320208117840558335 & $4.6(-14)$ & $2.6(-5)$ & $5.722687745(+1)$ & \\
\hline & 3 & -0.7285840464447930174399782 & $9.8(-41)$ & $3.4(-14)$ & $7.028215223(+4)$ & $7.028215223(+4)$ \\
\hline \multirow{4}{*}{$O M 1$} & 0 & -0.3 & $2.6(-1)$ & $4.3(-1)$ & & \\
\hline & 1 & -0.7302914840296724390574273 & $1.3(-8)$ & $1.7(-3)$ & $4.980739660(-2)$ & \\
\hline & 2 & -0.7285840464099897145888508 & $1.1(-31)$ & $3.5(-11)$ & 4.098844016 & \\
\hline & 3 & -0.7285840464448267167123331 & $5.3(-124)$ & $5.9(-42)$ & 4.027093448 & 4.027093448 \\
\hline \multirow{4}{*}{$O M 2$} & 0 & -0.3 & $2.6(-1)$ & $4.3(-1)$ & & \\
\hline & 1 & -0.7307723533603045420743033 & $2.7(-8)$ & $2.2(-3)$ & $6.355020749(-2)$ & \\
\hline & 2 & -0.7285840462868850837568170 & $1.0(-29)$ & $1.6(-10)$ & 6.887531010 & \\
\hline & 3 & -0.7285840464448267167123331 & $1.8(-115)$ & $4.1(-39)$ & 6.666147333 & 6.666147333 \\
\hline \multirow{4}{*}{$O M 3$} & 0 & -0.3 & $2.6(-1)$ & $4.3(-1)$ & & \\
\hline & 1 & -0.7307373761527420768440273 & $2.5(-8)$ & $2.2(-3)$ & $6.255475510(-2)$ & \\
\hline & 2 & -0.7285840463013044580708542 & $7.5(-30)$ & $1.4(-10)$ & 6.675395724 & \\
\hline & 3 & -0.7285840464448267167123331 & $5.3(-116)$ & $2.7(-39)$ & 6.466973456 & 6.466973456 \\
\hline \multirow{4}{*}{$O M 4$} & 0 & -0.3 & $2.6(-1)$ & $4.3(-1)$ & & \\
\hline & 1 & -0.7303054133977433599809357 & $1.3(-8)$ & $1.7(-3)$ & $5.020722652(-2)$ & \\
\hline & 2 & -0.7285840464088168705689866 & $1.2(-31)$ & $3.6(-11)$ & 4.101355219 & \\
\hline & 3 & -0.7285840464448267167123331 & $7.9(-124)$ & $6.8(-42)$ & 4.027093448 & 4.027093448 \\
\hline \multirow{4}{*}{$O M 5$} & 0 & -0.3 & $2.6(-1)$ & $4.5(-1)$ & & \\
\hline & 1 & -0.7482654918792924180501645 & $1.9(-5)$ & $2.0(-2)$ & $4.874526327(-1)$ & \\
\hline & 2 & -0.7285832914283975037429237 & $1.1(-18)$ & $7.6(-7)$ & 5.031086682 & \\
\hline & 3 & -0.7285840464448267167123318 & $5.7(-72)$ & $1.3(-24)$ & 4.027061336 & 4.027061336 \\
\hline
\end{tabular}

Table 4: Convergence behavior of different higher-order iterative methods on the test function $f_{3}(x)$

9. Li, S., Liao, X., Cheng, L.: A new fourth-order iterative method for finding multiple roots of nonlinear equations. Appl. Math. Comput. 215 (2009) 1288-1292. 


\begin{tabular}{|c|c|c|c|c|c|c|}
\hline Cases & $n$ & $x_{n}$ & $\left|f\left(x_{n}\right)\right|$ & $\left|x_{n+1}-x_{n}\right|$ & $\frac{x_{n+1}-x_{n}}{\left(x_{n}-x_{n-1}\right)^{4}}$ & $\eta$ \\
\hline \multirow{4}{*}{$H M T$} & 0 & 1 & $4.3(-19)$ & 1.0 & & \\
\hline & 1 & $7.828587819539840468767779(-7)$ & $3.1(-41)$ & $7.8(-7)$ & $7.828612334(-7)$ & $7.235152913(-7)$ \\
\hline & 2 & $2.717574459161567596486764(-31)$ & $2.0(-630)$ & $2.7(-31)$ & $7.235153361(-7)$ & \\
\hline & 3 & $3.946149396364377930545280(-129)$ & $3.4(-2587)$ & $3.9(-129)$ & $7.235152913(-7)$ & \\
\hline \multirow{4}{*}{$L C N$} & 0 & 1 & $4.3(-19)$ & 1.0 & & \\
\hline & 1 & $7.827308224966503817728439(-7)$ & $3.1(-141)$ & $7.8(-7)$ & $7.827332732(-7)$ & $7.234027376(-7)$ \\
\hline & 2 & $2.715375644700673086402099(-31)$ & $2.0(-630)$ & $2.7(-31)$ & $7.234027824(-7)$ & \\
\hline & 3 & $3.93278152676961481254200(-129)$ & $3.2(-2587)$ & $3.9(-129)$ & $7.234027376(-7)$ & \\
\hline \multirow{4}{*}{$S B S$} & 0 & 1 & $4.3(-19)$ & 1.0 & & \\
\hline & 1 & -0.008657778208344092130448359 & $2.3(-60)$ & $8.7(-3)$ & $8.364328644(-3)$ & $7.222994193(-7)$ \\
\hline & 2 & $4.294499850302673508998825(-13)$ & $1.9(-266)$ & $4.3(-13)$ & $7.643403163(-5)$ & \\
\hline & 3 & $2.456787724624677403816207(-56)$ & $2.6(-1131)$ & $2.5(-56)$ & $7.222994193(-7)$ & \\
\hline \multirow{4}{*}{$S B$} & 0 & 1 & $4.3(-19)$ & 1.0 & & \\
\hline & 1 & $7.813348181059581529335391(-7)$ & $3.0(-141)$ & $7.8(-7)$ & $7.813372600(-7)$ & $7.221778344(-7)$ \\
\hline & 2 & $2.691490759489867342829191(-31)$ & $1.6(-630)$ & $2.7(-31)$ & $7.221778790(-7)$ & \\
\hline & 3 & $3.789795075464724645722391(-129)$ & $1.5(-2587)$ & $3.8(-129)$ & $7.221778344(-7)$ & \\
\hline \multirow{4}{*}{$S B L$} & 0 & 1 & $4.3(-19)$ & 1.0 & & \\
\hline & 1 & $7.953057268795634055950551(-7)$ & $4.2(-141)$ & $8.0(-7)$ & $7.953082569(-7)$ & $7.344359844(-7)$ \\
\hline & 2 & $2.938261293075213048991021(-31)$ & $9.5(-630)$ & $2.9(-31)$ & $7.344360311(-7)$ & \\
\hline & 3 & $5.474136259993639201367362(-129)$ & $2.4(-2587)$ & $5.5(-129)$ & $7.344359844(-7)$ & \\
\hline \multirow{4}{*}{$Z C S$} & 0 & 1 & $4.3(-19)$ & 1.0 & & \\
\hline & 1 & $-6.637097701376525242035541(-8)$ & $1.1(-162)$ & $6.6(-8)$ & $6.637095941(-8)$ & $6.196562319(-8)$ \\
\hline & 2 & $2.097669799615884912384601(-17)$ & $1.1(-352)$ & $2.1(-17)$ & $1.080996486(-12)$ & \\
\hline & 3 & $-1.199773663528067231668232(-74)$ & $1.6(-1497)$ & $1.2(-74)$ & $6.196562319(-8)$ & \\
\hline \multirow{4}{*}{$Z M$} & 0 & 1 & $4.3(-19)$ & 1.0 & & \\
\hline & 1 & $7.828588035110395222651694(-7)$ & $3.1(-141)$ & $7.8(-7)$ & $7.828612550(-7)$ & $7.235153103(-7)$ \\
\hline & 2 & $2.717574829725725301121913(-31)$ & $2.0(-630)$ & $2.7(-31)$ & $7.235153551(-7)$ & \\
\hline & 3 & $3.946151652168463694980538(-129)$ & $3.4(-2587)$ & $3.9(-129)$ & $7.235153103(-7)$ & \\
\hline \multirow{4}{*}{$S A T Z 1$} & 0 & 1 & $4.3(-19)$ & 1.0 & & \\
\hline & 1 & $-4.536613753639024858789058(-5)$ & $5.6(-106)$ & $4.5(-5)$ & $4.535790612(-5)$ & \\
\hline & 2 & $3.949491101460006849782129(-18)$ & $3.5(-367)$ & $3.9(-18)$ & $9.324250216(-1)$ & \\
\hline & 3 & $-2.605976581155268321624286(-57)$ & $8.6(-1151)$ & $2.6(-57)$ & $1.071040639(+13)$ & $1.071040639(+13)$ \\
\hline \multirow{4}{*}{$S A T Z 2$} & 0 & 1 & $4.3(-19)$ & 1.0 & & \\
\hline & 1 & $-4.266898516948512437472135(-5)$ & $1.6(-106)$ & $4.3(-5)$ & $4.266170338(-5)$ & \\
\hline & 2 & $3.094789518979090264519079(-18)$ & $2.7(-269)$ & $3.1(-18)$ & $9.336451857(-1)$ & \\
\hline & 3 & $-1.180833833979428889742517(-57)$ & $1.1(-1157)$ & $1.2(-57)$ & $1.287254204(+13)$ & $1.287254204(+13)$ \\
\hline \multirow{4}{*}{$O M 1$} & 0 & 1 & $4.3(-19)$ & 1.0 & & \\
\hline & 1 & $-1.477021135487271075665121(-6)$ & $1.0(-135)$ & $1.5(-6)$ & $1.477012409(-6)$ & $1.264783617(-6)$ \\
\hline & 2 & $-6.019535391679813492511129(-30)$ & $1.6(-603)$ & $6.5(-30)$ & $1.264783329(-6)$ & \\
\hline & 3 & $-1.660611802498010944223058(-123)$ & $1.0(-2474)$ & $1.7(-123)$ & $1.264783617(-6)$ & \\
\hline \multirow{4}{*}{$O M 2$} & 0 & 1 & $4.3(-19)$ & 1.0 & & \\
\hline & 1 & $7.828727502589454827059765(-7)$ & $3.1(-141)$ & $7.8(-7)$ & $7.828752018(-7)$ & $7.235275807(-7)$ \\
\hline & 2 & $2.7178145827847979222793393(-31)$ & $2.0(-630)$ & $2.7(-31)$ & $7.235276255(-7)$ & \\
\hline & 3 & $3.947611352630203067255450(-129)$ & $3.5(-2587)$ & $3.9(-129)$ & $7.235275807(-7)$ & \\
\hline \multirow{4}{*}{$O M 3$} & 0 & 1 & $4.3(-19)$ & 1.0 & & \\
\hline & 1 & $7.827193013402466467601844(-7)$ & $3.1(-141)$ & $7.8(-7)$ & $7.827217519(-7)$ & $7.233926061(-7)$ \\
\hline & 2 & $2.715177748142894038690056(-31)$ & $1.9(-630)$ & $2.7(-31)$ & $7.233926508(-7)$ & \\
\hline & 3 & $3.931580103641188032260615(-129)$ & $3.2(-2587)$ & $3.9(-129)$ & $7.233926061(-7)$ & \\
\hline \multirow{4}{*}{$O M 4$} & 0 & 1 & $4.3(-19)$ & 1.0 & & \\
\hline & 1 & $-1.477123319694082282842075(-6)$ & $1.0(-135)$ & $1.5(-6)$ & $1.477114592(-6)$ & $1.264783617(-6)$ \\
\hline & 2 & $-6.021201353013852867532080(-30)$ & $1.6(-603)$ & $6.0(-30)$ & $1.264783329(-6)$ & \\
\hline & 3 & $-1.662450923674687122831356(-123)$ & $1.1(-2474)$ & $1.7(-123)$ & $1.264783617(-6)$ & \\
\hline \multirow{4}{*}{$O M 5$} & 0 & 1 & $4.3(-19)$ & 1.0 & & \\
\hline & 1 & $-1.477123319694082282842075(-6)$ & $1.0(-135)$ & $1.5(-6)$ & $1.477114592(-6)$ & $1.264783617(-6)$ \\
\hline & 2 & $-6.021201353013852867532080(-30)$ & $1.6(-603)$ & $6.0(-30)$ & $1.264783329(-6)$ & \\
\hline & 3 & $-1.662450923674687122831356(-123)$ & $1.1(-2474)$ & $1.7(-123)$ & $1.264783617(-6)$ & \\
\hline
\end{tabular}

Table 5: Convergence behavior of different higher-order iterative methods on the test function $f_{4}(x)$. 


\begin{tabular}{|c|c|c|c|c|c|c|}
\hline Cases & $n$ & $x_{n}$ & $\left|f\left(x_{n}\right)\right|$ & $\left|x_{n+1}-x_{n}\right|$ & \begin{tabular}{|l|}
$x_{n+1}-x_{n}$ \\
$\left(x_{n}-x_{n-1}\right)^{4}$
\end{tabular} & $\eta$ \\
\hline \multirow{4}{*}{$H M T$} & 0 & 1.8 & $2.0(-4)$ & $4.7(-1)$ & & \multirow{4}{*}{$1.504525186(+4)$} \\
\hline & 1 & 1.752635974832068545325330 & $2.3(-7)$ & $2.6(-3)$ & $5.236740581(+2)$ & \\
\hline & 2 & 1.750000520632870976970862 & $8.1(-15)$ & $5.2(-7)$ & $1.079219272(+4)$ & \\
\hline & 3 & 1.750000000000000000001105 & $3.7(-44)$ & $1.1(-21)$ & $1.504525186(+4)$ & \\
\hline \multirow{4}{*}{$L C N$} & 0 & 1.8 & $2.0(-4)$ & $4.7(-1)$ & & \multirow{4}{*}{$1.388813078(+4)$} \\
\hline & 1 & 1.752523213564796124343964 & $2.1(-7)$ & $2.5(-3)$ & $4.965446191(+2)$ & \\
\hline & 2 & 1.750000409392763577418605 & $5.0(-15)$ & $4.1(-7)$ & $1.010662146(+4)$ & \\
\hline & 3 & 1.750000000000000000000390 & $4.6(-45)$ & $3.9(-22)$ & $1.388813078(+4)$ & \\
\hline \multirow{4}{*}{$S B S$} & 0 & 1.8 & $2.0(-4)$ & $4.7(-1)$ & & \multirow{4}{*}{$1.263474539(-2)$} \\
\hline & 1 & 1.752638728771643002591608 & $2.3(-7)$ & $2.6(-3)$ & $3.990317988(-1)$ & \\
\hline & 2 & 1.750000520807595366165174 & $8.1(-15)$ & $5.2(-7)$ & $1.166565670(-2)$ & \\
\hline & 3 & 1.750000000000000000001101 & $3.6(-44)$ & $1.1(-21)$ & $1.263474539(-2)$ & \\
\hline \multirow{4}{*}{$S B$} & 0 & 1.8 & $2.0(-4)$ & $4.7(-1)$ & & \multirow{4}{*}{$1.388813078(+4)$} \\
\hline & 1 & 1.752523213564796124343964 & $2.1(-7)$ & $2.5(-3)$ & $4.965446191(+2)$ & \\
\hline & 2 & 1.750000409392763577418605 & $5.0(-15)$ & $4.1(-7)$ & $1.010662146(+4)$ & \\
\hline & 3 & 1.750000000000000000000390 & $4.6(-45)$ & $3.9(-22)$ & $1.388813078(+4)$ & \\
\hline \multirow{4}{*}{$S B L$} & 0 & 1.8 & $2.0(-4)$ & $4.7(-1)$ & & \multirow{4}{*}{$1.276646091(-2)$} \\
\hline & 1 & 1.752523213564796124343964 & $2.1(-7)$ & $2.5(-3)$ & $4.355919298(-2)$ & \\
\hline & 2 & 1.750000409392763577418605 & $5.0(-15)$ & $4.1(-7)$ & $1.267632710(-2)$ & \\
\hline & 3 & 1.750000000000000000000390 & $4.6(-45)$ & $3.9(-22)$ & $1.276646091(-2)$ & \\
\hline \multirow{4}{*}{$Z C S$} & 0 & 1.8 & $2.0(-4)$ & $4.7(-1)$ & & \multirow{4}{*}{$2.545702314(+4)$} \\
\hline & 1 & 1.753077732689054788153803 & $3.1(-7)$ & $3.1(-3)$ & $6.346171766(+2)$ & \\
\hline & 2 & 1.750001439104082535103692 & $6.2(-14)$ & $1.4(-6)$ & $1.606871270(+4)$ & \\
\hline & 3 & 1.750000000000000000109188 & $3.6(-40)$ & $1.1(-19)$ & $2.545702314(+4)$ & \\
\hline \multirow{4}{*}{$Z M$} & 0 & 1.8 & $2.0(-4)$ & $4.7(-1)$ & & \multirow{4}{*}{$1.280504115(-2)$} \\
\hline & 1 & 1.752726454151483442238463 & $2.4(-7)$ & $2.7(-3)$ & $4.381618269(-2)$ & \\
\hline & 2 & 1.750000632399803226474600 & $1.2(-14)$ & $6.3(-7)$ & $1.271385203(-2)$ & \\
\hline & 3 & 1.750000000000000000002591 & $2.0(-43)$ & $2.6(-21)$ & $1.280504115(-2)$ & \\
\hline \multirow{4}{*}{$S A T Z 1$} & 0 & 1.8 & $2.0(-4)$ & $4.4(-2)$ & & \multirow{4}{*}{$9.012610049(+6)$} \\
\hline & 1 & 1.755680568432913874922503 & $1.2(-6)$ & $5.6(-3)$ & $1.455629472(+3)$ & \\
\hline & 2 & 1.750064562388692565624633 & $1.3(-10)$ & $6.5(-4)$ & $6.490352292(+4)$ & \\
\hline & 3 & 1.750000000156590253742911 & $7.4(-22)$ & $1.6(-10)$ & $9.012610049(+6)$ & \\
\hline \multirow{4}{*}{$S A T Z 2$} & 0 & 1.8 & $2.0(-4)$ & $4.6(-2)$ & & \\
\hline & 1 & 1.753830359714441037125345 & $5.0(-7)$ & $3.8(-3)$ & $1.477114592(-6)$ & $1.264783617(-6)$ \\
\hline & 2 & 1.750009383638041526135206 & $2.6(-12)$ & $9.4(-6)$ & $1.264783329(-6)$ & \\
\hline & 3 & 1.750000000000161402863588 & $7.8(-28)$ & $1.6(-13)$ & $1.264783617(-6)$ & \\
\hline & 0 & 1.8 & $2.0(-4)$ & $4.8(-2)$ & & \\
\hline & 1 & 1.751713796508416879598061 & $9.3(-8)$ & $6.4(-9)$ & $3.152574793(+2)$ & $3.439690471(-3)$ \\
\hline$O M 1$ & 2 & 1.750000006368346428936612 & $1.2(-18)$ & $6.4(-9)$ & $7.382375318(+21)$ & \\
\hline & 3 & 1.750000000000000000000000 & $9.6(-73)$ & $5.7(-36)$ & $3.439690471(-3)$ & \\
\hline & 0 & 1.8 & $2.0(-4)$ & $4.7(-2)$ & & \\
\hline & 1 & 1.752889533818870707034275 & $2.7(-7)$ & $2.9(-3)$ & $5.864419084(+2)$ & $1.851626283(+4)$ \\
\hline$O M 2$ & 2 & 1.750000885938106677419627 & $2.4(-14)$ & $8.9(-7)$ & $1.272403807(+4)$ & \\
\hline & 3 & 1.750000000000000000011407 & $3.9(-42)$ & $1.1(-20)$ & $1.851626283(+4)$ & \\
\hline & 0 & 1.8 & $2.0(-4)$ & $4.7(-1)$ & & \\
\hline$O M 3$ & 1 & 1.752523213564796124343964 & $2.1(-7)$ & $2.5(-3)$ & $4.296799492(-2)$ & $1.265072016(-2)$ \\
\hline 0103 & 2 & 1.750000409392763577418605 & $5.0(-15)$ & $4.1(-7)$ & $1.256323789(-2)$ & \\
\hline & 3 & 1.750000000000000000000390 & $4.6(-45)$ & $3.9(-22)$ & $1.265072016(-2)$ & \\
\hline & 0 & 1.8 & $2.0(-4)$ & $4.7(-2)$ & & \\
\hline & 1 & 1.751459743679674885454601 & $6.7(-8)$ & $1.5(-3)$ & $2.629467992(+2)$ & $3.823958268(+4)$ \\
\hline$O M 4$ & 2 & 1.750000001524876992581441 & $7.0(-20)$ & $1.5(-9)$ & $3.358382919(+2)$ & \\
\hline & 3 & 1.750000000000000000000000 & $1.3(-79)$ & $2.1(-39)$ & $3.823958268(+4)$ & \\
\hline & 0 & 1.8 & $2.0(-4)$ & $4.9(-2)$ & & \\
\hline & 1 & 1.751459740956958601485950 & $6.7(-8)$ & $1.5(-3)$ & $2.629462497(+2)$ & $3823923339(+4)$ \\
\hline$O M 5$ & 2 & 1.750000001524863069277435 & $7.0(-20)$ & $1.5(-9)$ & $3.358377310(+2)$ & \\
\hline & 3 & 1.750000000000000000000000 & $1.3(-79)$ & $2.1(-39)$ & $3.823923339(+4)$ & \\
\hline
\end{tabular}

Table 6: Convergence behavior of different higher-order iterative methods on the test function $f_{5}(x)$. 


\begin{tabular}{|c|c|c|c|c|c|c|}
\hline Cases & $n$ & $x_{n}$ & $\left|f\left(x_{n}\right)\right|$ & $\left|x_{n+1}-x_{n}\right|$ & $\frac{x_{n+1}-x_{n}}{\left(x_{n}-x_{n-1}\right)^{4}}$ & $\eta$ \\
\hline \multirow{4}{*}{$H M T$} & 0 & 0.06 & 6.0 & $5.0(-3)$ & & \multirow{4}{*}{$7.450530186(-2)$} \\
\hline & 1 & 0.05504622446911478013103692 & $5.4(-8)$ & $4.4(-11)$ & $7.345821216(-2)$ & \\
\hline & 2 & 0.05504622451335177827483421 & $3.5(-40)$ & $2.9(-43)$ & $7.345821216(-2)$ & \\
\hline & 3 & 0.05504622451335177827483421 & $6.0(-169)$ & $4.9(-172)$ & $7.450530186(-2)$ & \\
\hline \multirow{4}{*}{$L C N$} & 0 & 0.06 & 6.0 & $5.0(-3)$ & & \multirow{4}{*}{$3.320288128(-)$} \\
\hline & 1 & 0.05504622471136734450357120 & $2.4(-7)$ & $2.0(-10)$ & $3.288168938(-1)$ & \\
\hline & 2 & 0.05504622451335177827483421 & $6.2(-37)$ & $5.1(-40)$ & $3.320288127(-1)$ & \\
\hline & 3 & 0.05504622451335177827483421 & $2.7(-155)$ & $2.3(-158)$ & $3.320288128(-11)$ & \\
\hline \multirow{4}{*}{$S B S$} & 0 & 0.06 & 6.0 & $5.0(-3)$ & & \multirow{4}{*}{$7.04399607(-2)$} \\
\hline & 1 & 0.05504622446916535317059648 & $5.4(-8)$ & $4.4(-11)$ & $7.337423258(-2)$ & \\
\hline & 2 & 0.05504622451335177827483421 & $3.3(-40)$ & $2.7(-43)$ & $7.043996068(-2)$ & \\
\hline & 3 & 0.05504622451335177827483421 & $4.4(-169)$ & $3.7(-172)$ & $7.04399607(-2)$ & \\
\hline \multirow{4}{*}{$S B$} & 0 & 0.06 & 6.0 & $5.0(-3)$ & & \multirow{4}{*}{$2.588526722(-1)$} \\
\hline & 1 & 0.05504622466828950617089380 & $1.9(-7)$ & $1.5(-10)$ & $2.572835137(-1)$ & \\
\hline & 2 & 0.05504622451335177827483421 & $1.8(-37)$ & $1.5(-40)$ & $2.588526721(-1)$ & \\
\hline & 3 & 0.05504622451335177827483421 & $1.6(-157)$ & $1.3(-160)$ & $2.588526722(-1)$ & \\
\hline \multirow{4}{*}{$S B L$} & 0 & 0.06 & 6.0 & $5.0(-3)$ & & \multirow{4}{*}{$2.736179928(-3)$} \\
\hline & 1 & 0.05504622451504802142669122 & $2.1(-9)$ & $1.7(-12)$ & $2.816714497(-3)$ & \\
\hline & 2 & 0.05504622451335177827483421 & $2.7(-47)$ & $2.3(-50)$ & $2.736179928(-3)$ & \\
\hline & 3 & 0.05504622451335177827483421 & $8.7(-199)$ & $7.2(-202)$ & $2.736179928(-3)$ & \\
\hline \multirow{4}{*}{$Z C S$} & 0 & 0.06 & 6.0 & $5.0(-3)$ & & \multirow{4}{*}{$4.741058894(-4)$} \\
\hline & 1 & 0.05504622479639067875813967 & $3.7(-4)$ & $2.8(-10)$ & $4.700033449(-1)$ & \\
\hline & 2 & 0.05504622451335177827483421 & $3.7(-36)$ & $3.0(-39)$ & $4.741058891(-1)$ & \\
\hline & 3 & 0.05504622451335177827483421 & $4.9(-152)$ & $4.1(-155)$ & $4.741058894(-4)$ & \\
\hline \multirow{4}{*}{$Z M$} & 0 & 0.06 & 6.0 & $5.0(-3)$ & & \multirow{4}{*}{$1.85676531(-1)$} \\
\hline & 1 & 0.05504622462459516774941427 & $1.3(-7)$ & $1.1(-10)$ & $1.847264027(-1)$ & \\
\hline & 2 & 0.05504622451335177827483421 & $3.4(-38)$ & $2.8(-41)$ & $1.85676531(-1)$ & \\
\hline & 3 & 0.05504622451335177827483421 & $1.5(-160)$ & $1.2(-163)$ & $1.85676531(-1)$ & \\
\hline \multirow{4}{*}{$S A T Z 1$} & 0 & 0.06 & 6.0 & $5.0(-3)$ & & \multirow{4}{*}{$6.827507706(+22)$} \\
\hline & 1 & 0.05504624898026988270253543 & $3.0(-5)$ & $2.4(-8)$ & $4.062960212(+1)$ & \\
\hline & 2 & 0.05504622451335177827483716 & $3.6(-21)$ & $3.0(-24)$ & $8.241081365(+6)$ & \\
\hline & 3 & 0.05504622451335177827483421 & $6.3(-69)$ & $5.2(-72)$ & $6.827507706(+22)$ & \\
\hline \multirow{4}{*}{$S A T Z 2$} & 0 & 0.06 & 6.0 & $5.0(-3)$ & & \\
\hline & 1 & 0.05504620943399314696361921 & $1.8(-5)$ & $1.5(-8)$ & $2.503988345(+1)$ & $2.916428788(+23)$ \\
\hline & 2 & 0.05504622451335177827483464 & $5.2(-22)$ & $4.3(-25)$ & $8.307994319(+6)$ & \\
\hline & 3 & 0.05504622451335177827483421 & $1.2(-71)$ & $9.9(-75)$ & $2.916428788(+23)$ & \\
\hline & 0 & 0.06 & 6.0 & $5.0(-3)$ & & \\
\hline$O M 1$ & 1 & 0.05504622447305622400664137 & $4.9(-8)$ & $4.0(-11)$ & $6.691320633(-2)$ & $7.043996071(-2)$ \\
\hline ONI & 2 & 0.05504622451335177827483421 & $2.3(-40)$ & $1.9(-43)$ & $7.043996074(-2)$ & \\
\hline & 3 & 0.05504622451335177827483421 & $1.0(-169)$ & $8.4(-173)$ & $7.043996071(-2)$ & \\
\hline & 0 & 0.06 & 6.0 & $5.0(-3)$ & & \\
\hline רגומת & 1 & 0.05504622479895957925655606 & $3.5(-7)$ & $2.9(-10)$ & $4.742691618(-1)$ & $4.783810941(-1)$ \\
\hline ONI & 2 & 0.05504622451335177827483421 & $3.9(-36)$ & $3.2(-39)$ & $4.783810938(-1)$ & \\
\hline & 3 & 0.05504622451335177827483421 & $6.0(-152)$ & $4.9(-155)$ & $4.783810941(-1)$ & \\
\hline & 0 & 0.06 & 6.0 & $5.0(-3)$ & & \\
\hline ב & 1 & 0.05504622453699903953651134 & $2.9(-8)$ & $2.4(-11)$ & $3.926771036(-2)$ & $3.932425025(-2)$ \\
\hline ONI & 2 & 0.05504622451335177827483421 & $1.5(-41)$ & $1.2(-44)$ & $3.932425025(-2)$ & \\
\hline & 3 & 0.05504622451335177827483421 & $1.1(-174)$ & $9.0(-178)$ & $3.932425025(-2)$ & \\
\hline & 0 & 0.06 & 6.0 & $5.0(-3)$ & & \\
\hline$O M 4$ & 1 & 0.05504622447154416118757406 & $5.1(-8)$ & $4.2(-11)$ & $6.942407806(-2)$ & $1.7043996071(-2)$ \\
\hline ONI & 2 & 0.05504622451335177827483421 & $2.6(-40)$ & $2.2(-43)$ & $7.043996072(-2)$ & \\
\hline & 3 & 0.05504622451335177827483421 & $1.8(-169)$ & $1.5(-172)$ & $1.7043996071(-2)$ & \\
\hline & 0 & 0.06 & 6.0 & $4.9(-3)$ & & \\
\hline$O M 5$ & 1 & 0.05509861525531043184190827 & $6.4(-2)$ & $5.2(-5)$ & $9.077763231(+4)$ & $6.953744528(+12)$ \\
\hline ONI & 2 & 0.05504622457595169612771086 & $7.6(-8)$ & $6.3(-11)$ & $8.309169974(+6)$ & \\
\hline & 3 & 0.05504622451335177827483421 & $1.3(-25)$ & $1.1(-28)$ & $6.953744528(+12)$ & \\
\hline
\end{tabular}

Table 7: Convergence behavior of different higher-order iterative methods on the test function $f_{6}(x)$. 


\begin{tabular}{|llllll|}
\hline Cases & Exp.1 & Exp.2 & Exp.3 & Exp.4 & Exp.5 \\
\hline$H M T$ & 0.4898 & 0.144 & 1.145 & 0.895 & 0.705 \\
$L C N$ & 0.3966 & 0.1438 & 0.783 & 0.627 & 0.5684 \\
$S B S$ & 0.5676 & 0.143 & 1.061 & 0.983 & 0.7486 \\
$S B$ & 0.3652 & 0.1434 & 0.6802 & 0.5646 & 0.4836 \\
$S B L$ & 0.4148 & 0.1622 & 0.7706 & 0.6308 & 0.5306 \\
$Z C S$ & 0.58 & 0.156 & 0.9924 & 0.6492 & 0.652 \\
$Z M$ & 0.5114 & 0.15 & 0.8734 & 0.674 & 0.5458 \\
$S A T Z 1$ & 0.5022 & 0.1562 & 0.6808 & 0.8112 & 0.7896 \\
$S A T Z 2$ & 0.5088 & 0.1434 & 0.7088 & 0.8362 & 0.8114 \\
$O M 1$ & 0.3274 & 0.1596 & 0.7736 & 0.7364 & 0.4054 \\
$O M 2$ & 0.3443 & 0.1498 & 0.939 & 0.7304 & 0.621 \\
$O M 3$ & 0.3806 & 0.1497 & 0.6518 & 0.6086 & 0.5586 \\
$O M 4$ & 0.4276 & 0.1374 & 0.974 & 0.7428 & 0.4114 \\
$O M 5$ & 0.4618 & 0.125 & 1.008 & 0.8144 & 0.6804 \\
\hline
\end{tabular}

Table 8: CPU Time

10. Petković, M.S., Neta, B., Petković, L.D., Džunić, J.: Multipoint methods for solving nonlinear equations, Academic Press 2013.

11. Sbibih, D., Serghini, A., Tijini, A., Zidna, A., A general family of third order method for finding multiple roots, AMC 233 (2014) 338-350.

12. Schröder, E: Über unendlichviele Algorithm zur Auffosung der Gleichungen. Math. Annal. 2 (1870) 317-365.

13. Sharifi, M., Babajee, D.K.R., Soleymani, F.: Finding the solution of nonlinear equations by a class of optimal methods. Comput. Math. Appl. 63 (2012) 764-774.

14. Soleymani, F., Babajee, D.K.R.: Computing multiple zeros using a class of quartically convergent methods. Alex. Eng. J. 52 (2013) 531-541.

15. Soleymani, F., Babajee, D.K.R., Lofti, T.: On a numerical technique forfinding multiple zeros and its dynamic. J. Egypt. Math. Soc. 21 (2013) $346-353$.

16. Traub, J.F., Iterative Methods for the Solution of Equations. Prentice-Hall, Englewood Cliffs, NJ 1964.

17. Zhou, X., Chen, X., Song, Y, Families of third and fourth order methods for multiple roots of nonlinear equations. Appl. Math. Comput. 219 (2013) 6030-6038.

18. Zhou, X., Chen, X., Song, Y.: Constructing higher-order methods for obtaining the muliplte roots of nonlinear equations. J. Comput. Math. Appl. 235 (2011) 4199-4206. 


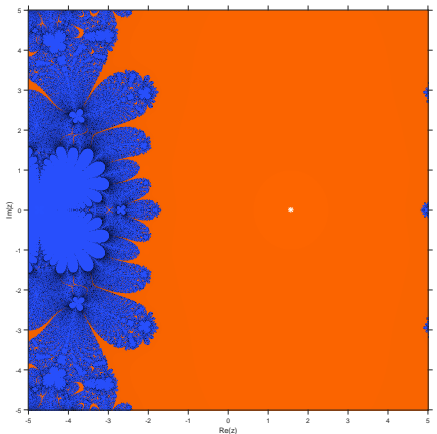

(a) HMT

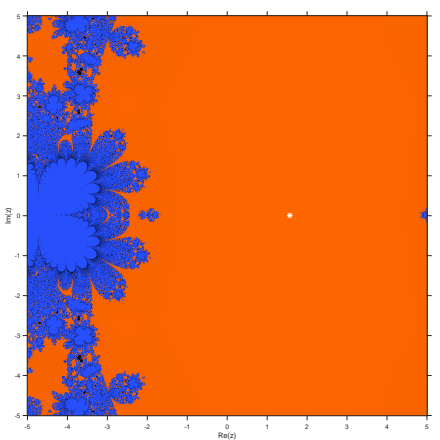

(d) SB

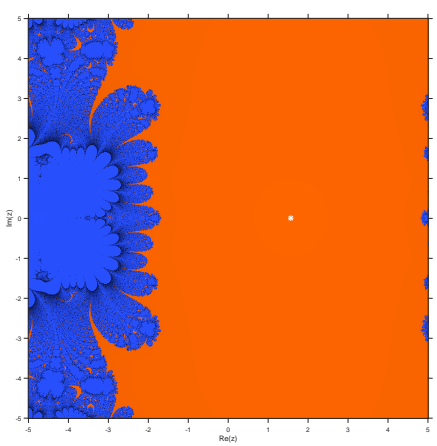

(g) ZM

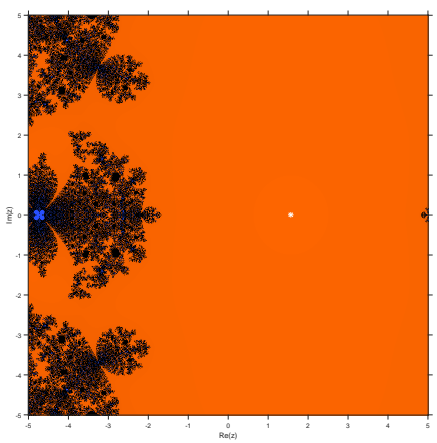

(j) OM3

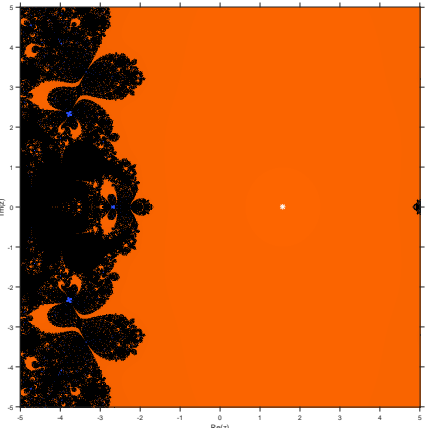

(b) $\mathrm{LCN}$

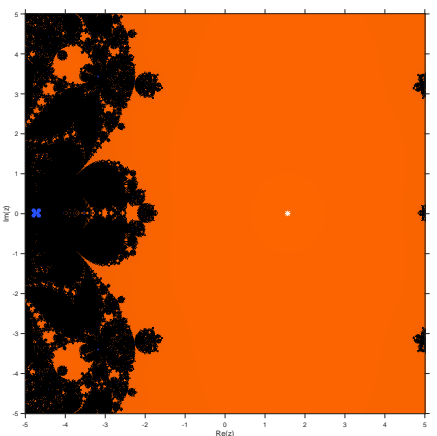

(e) SBL

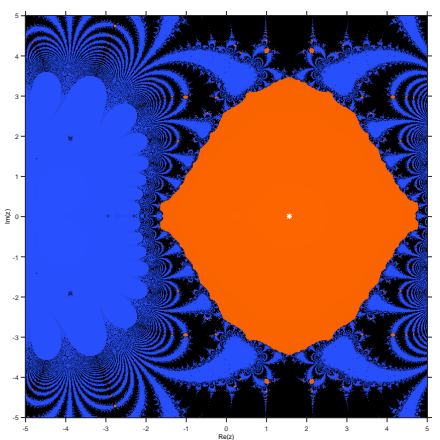

(h) OM1

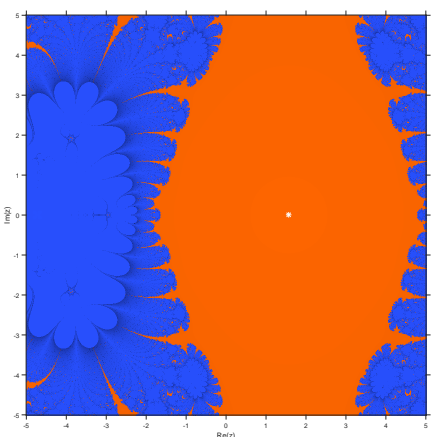

(k) OM4

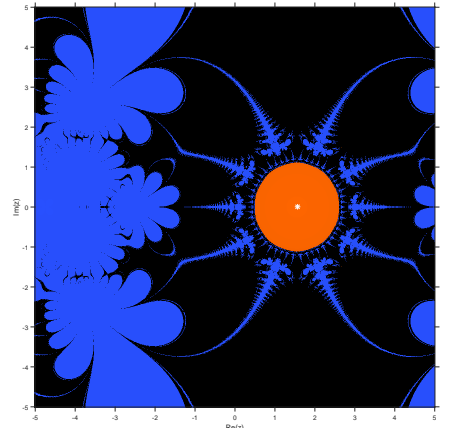

(c) SBS

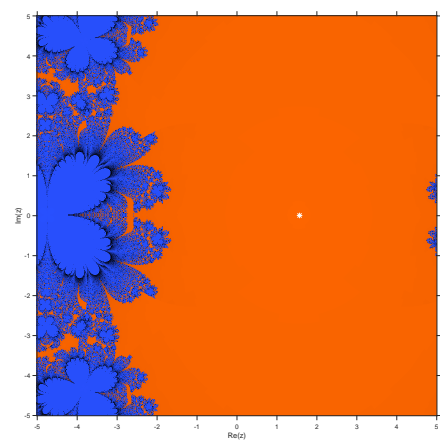

(f) ZCS

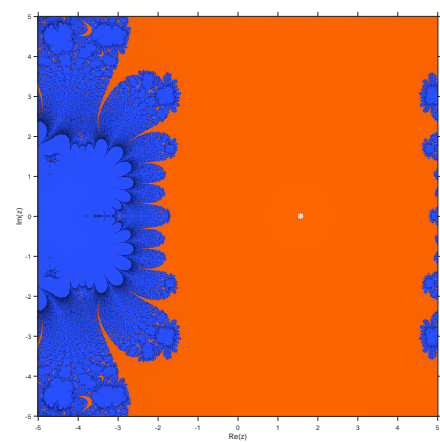

(i) $\mathrm{OM} 2$

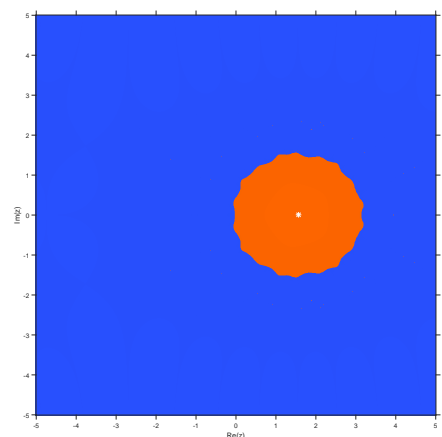

(1) OM5

Fig. 10: Dynamical planes of the different methods on test function $f_{1}(x)$ 


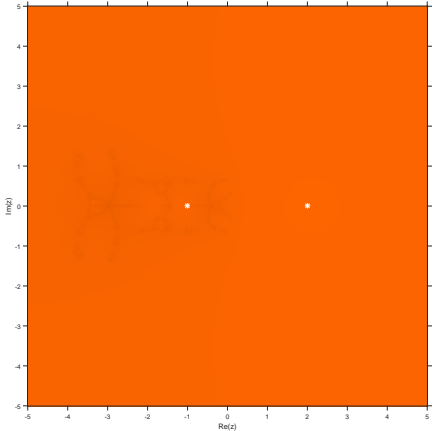

(a) HMT

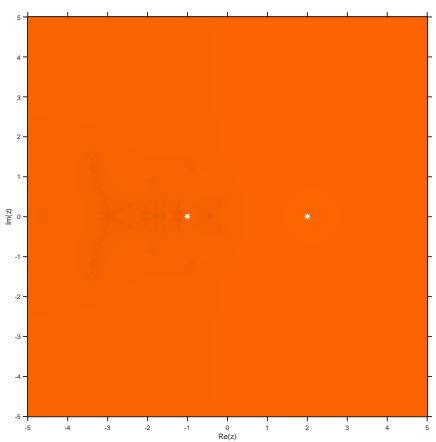

(d) SB

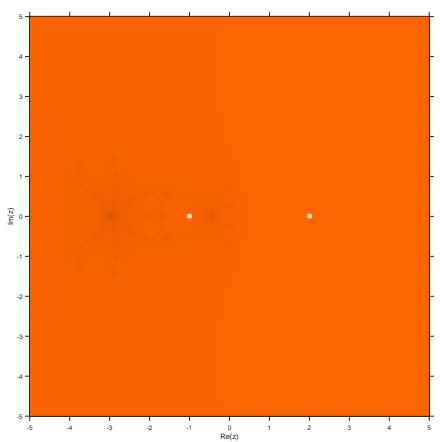

(g) ZM

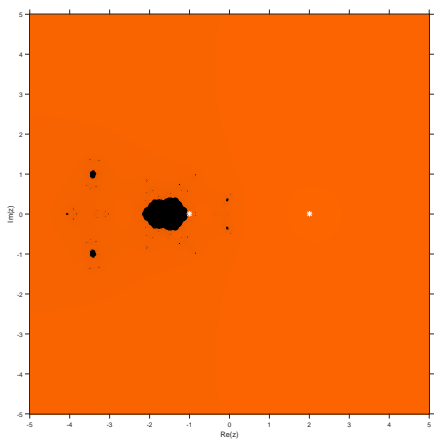

(j) OM3

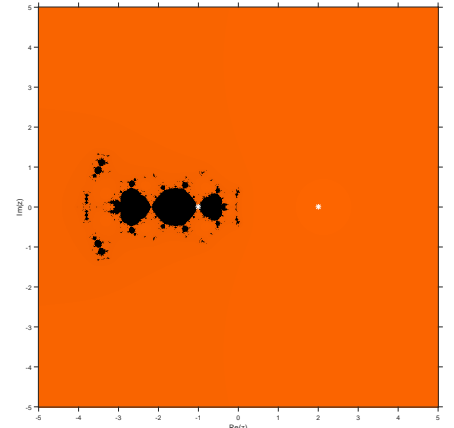

(b) LCN

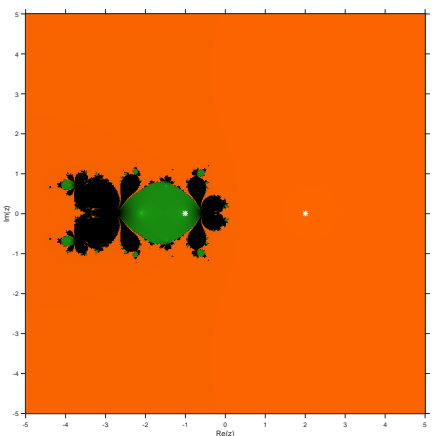

(e) SBL

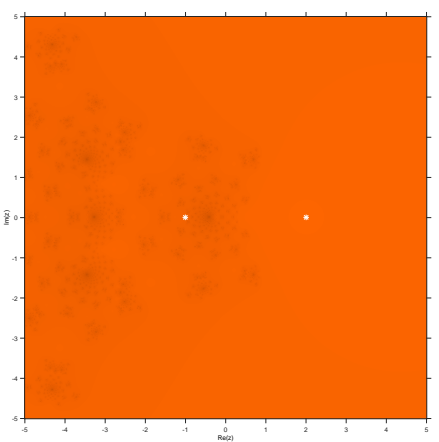

(h) OM1

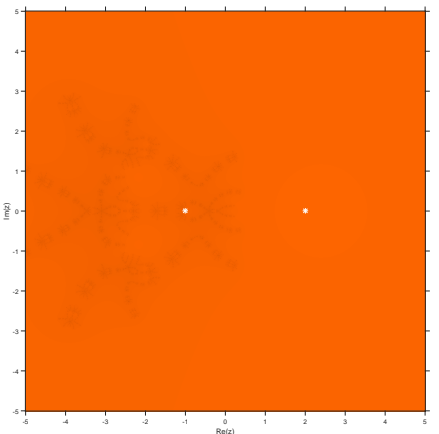

(k) OM4

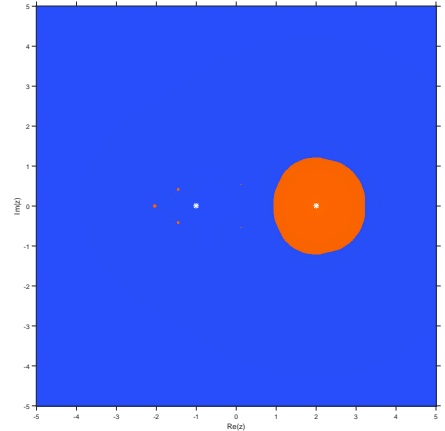

(c) SBS

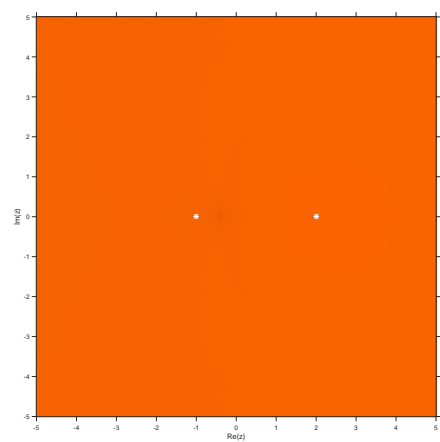

(f) ZCS

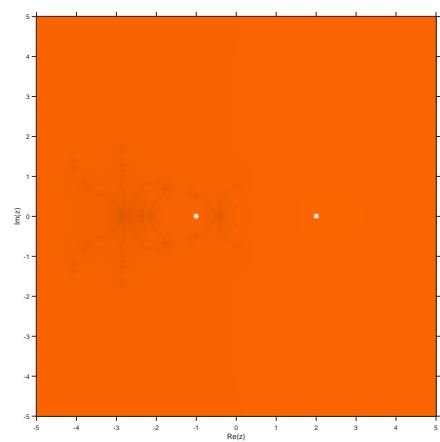

(i) OM2

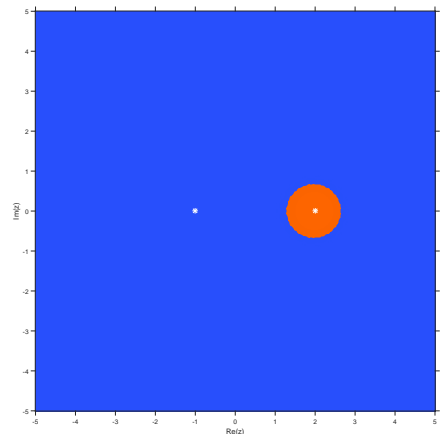

(1) OM5

Fig. 11: Dynamical planes of the different methods on test function $f_{2}(x)$ 